\title{
PARACONTROLLED QUASILINEAR SPDES
}

\author{
By MARCO Furlan AND MASSIMILIANO GUBINELli
}

\section{PSL-Université Paris-Dauphine and IAM \& HCM, Universität Bonn}

We introduce a nonlinear paracontrolled calculus and use it to renormalise a class of singular SPDEs including certain quasilinear variants of the periodic two-dimensional parabolic Anderson model.

1. Introduction. We show how to renormalise a class of general quasilinear equations of which one of the simplest examples is the following parabolic SPDE:

$$
\begin{aligned}
\partial_{t} u(t, x)-a(u(t, x)) \Delta u(t, x) & =\xi(x), \\
u(0, x) & =u_{0}(x), \quad x \in \mathbb{T}^{2}, t \geq 0,
\end{aligned}
$$

with $a: \mathbb{R} \rightarrow[\lambda, 1]$ for $\lambda>0$ a uniformly bounded $C^{3}$ diffusion coefficient, and $\left\|a^{(k)}\right\|_{L^{\infty}} \leq 1$ for $k=0, \ldots, 3$. We assume that $\xi \in \mathscr{C}^{\alpha-2}\left(\mathbb{T}^{2}\right)$ with $2 / 3<\alpha<1$ where $\mathscr{C}^{\alpha}\left(\mathbb{T}^{2}\right)$ is the Besov space $B_{\infty, \infty}^{\alpha}\left(\mathbb{T}^{2}\right)$. This would apply, for example, to the space white noise on $\mathbb{T}^{2}$. In this case, we only expect that $u(t, \cdot) \in \mathscr{C}^{\alpha}\left(\mathbb{T}^{2}\right)$ and the term $a(u(t, \cdot)) \Delta u(t, \cdot)$ is not well defined when $2 \alpha-2<0$. Equation (1) is a quasilinear generalisation of the two-dimensional periodic parabolic Anderson model (PAM). The linear parabolic Anderson model has a relatively straightforward treatment with the standard paracontrolled calculus by Gubinelli, Imkeller and Perkowski (2015): the choice of a nonlinear variant of this model is motivated by the intention of putting in evidence the main ideas of our technique in the simplest possible setting, without being burdened in technicalities.

Let us remark from the beginning that the framework developed in this paper allows us to deal as well with a class of equations of the form

$$
\begin{aligned}
& a_{3}(u(t, x)) \partial_{t} u(t, x)-a_{1}(u(t, x)) \Delta u(t, x) \\
& \quad=\xi\left(a_{2}(u(t, x)), t, x\right), \quad x \in \mathbb{T}^{2}, t \geq 0,
\end{aligned}
$$

where $a_{1}, a_{2}, a_{3}$ are sufficiently smooth nondegenerate coefficients and $\xi(z, t, x)$ is a Gaussian process with covariance

$$
\begin{aligned}
& \mathbb{E}\left[\xi(z, t, x) \xi\left(z^{\prime}, t^{\prime}, x^{\prime}\right)\right] \\
& \quad=F\left(z, z^{\prime}\right) Q\left(t-t^{\prime}, x-x^{\prime}\right), \quad x, x^{\prime} \in \mathbb{T}^{2}, t, t^{\prime}, z, z^{\prime} \in \mathbb{R},
\end{aligned}
$$

Received November 2016; revised April 2018.

MSC2010 subject classifications. 60H15, 35S50.

Key words and phrases. Quasilinear SPDEs, paracontrolled distributions, paraproducts. 
with $F$ a smooth function and $Q$ a distribution of parabolic regularity $\rho>-4 / 3$. This includes as a special case the space white noise discussed before, but we could consider a time white noise with a regular dependence on the space variable, or some noise which is mildly irregular in space and time.

Moreover, the scalar character of the equation or of the nonlinear diffusion coefficient will not play any specific role and we could consider vector-valued equations with general diffusion coefficients, provided the template problem (6) below is uniformly parabolic.

For the sake of clarity and simplicity, we will discuss mainly the basic example (1) since this contains already most of the technical difficulties. The fact that one can handle models as general as (2) can be seen as a direct byproduct of the techniques we will introduce below. Let us state one simple result that can be obtained via the theory developed in this paper.

THEOREM 1.1. Fix $2 / 3<\alpha<1$. Let $\xi \in \mathscr{C}^{\alpha-2}\left(\mathbb{T}^{2}\right)$ be a space white noise with zero average on the torus, $u_{0} \in \mathscr{C}^{\alpha}$ an initial condition and $a: \mathbb{R} \rightarrow[\lambda, 1]$ for some $\lambda>0, a \in C^{3}(\mathbb{R})$ and $\left\|a^{(k)}\right\|_{L^{\infty}} \leq 1 \forall k \in 0, \ldots, 3$. Let $\left(\xi_{\varepsilon}, u_{0, \varepsilon}\right)_{\varepsilon>0}$ be a family of smooth approximations of $\xi, u_{0}$ obtained by convolution with a rescaled smoothing kernel and $u_{\varepsilon}$ the classical solution to the Cauchy problem

$$
\partial_{t} u_{\varepsilon}-a\left(u_{\varepsilon}\right) \Delta u_{\varepsilon}=\xi_{\varepsilon}+\sigma_{\varepsilon} \frac{a^{\prime}\left(u_{\varepsilon}\right)}{a\left(u_{\varepsilon}\right)^{2}}, \quad u(0)=u_{0, \varepsilon} .
$$

Then we can choose a sequence of constants $\left(\sigma_{\varepsilon}\right)_{\varepsilon>0}$ and a random time $T>0$ in such a way that the family of r.v. $\left(u_{\varepsilon}\right)_{\varepsilon>0} \subseteq \mathscr{L}_{T}^{\alpha}\left(\mathbb{T}^{2}\right)$ converges almost surely as $\varepsilon \rightarrow 0$ to a random element $u \in \mathscr{L}_{T}^{\alpha}\left(\mathbb{T}^{2}\right)$, where $\mathscr{L}_{T}^{\alpha}$ is the parabolic space $C\left([0, T], \mathscr{C}^{\alpha}\left(\mathbb{T}^{2}\right)\right) \cap C^{\alpha / 2}\left([0, T], \mathscr{C}^{0}\left(\mathbb{T}^{2}\right)\right)$.

This element can be characterised as the solution to a paracontrolled singular SPDE (see below for more details).

In order to devise a suitable formulation of equation (1) and obtain a theory with $u \in \mathscr{C}^{\alpha}$ we decompose the nonlinear diffusion term in the 1.h.s. with the help of Bony's paraproduct Meyer (1980) and write

$$
\partial_{t} u-a(u) \prec \Delta u=\xi+\Phi(u)
$$

with

$$
\Phi(u):=a(u) \circ \Delta u+a(u) \succ \Delta u,
$$

where $\prec, \succ$ are standard paraproducts and $\circ$ denotes the resonant product (see the Appendix for the definitions). In (4), the 1.h.s. is always well defined, irrespectively of the regularity of the function $u$, and the problem becomes that of controlling the resonant product $a(u) \circ \Delta u$ appearing in the r.h.s. A key point of the analysis put forward below is that this term can be expected to be of regularity $2 \alpha-2>\alpha-2$, better than the leading term $\xi \in \mathscr{C}^{\alpha-2}$. 
Our approach can be described as follows. For an equation of the form

$$
\partial_{t} u-a_{1}(u) \Delta u=a_{2}(u) \xi,
$$

we consider at first a parametric "template" problem with constant coefficients $\eta_{1}$, $\eta_{2}$,

$$
\partial_{t} \vartheta(\eta, t, x)-\eta_{1} \Delta \vartheta(\eta, t, x)=\eta_{2} \xi(t, x),
$$

where now $\eta=\left(\eta_{1}, \eta_{2}\right)$ is a fixed vector in $\mathbb{R}^{2}$. A nonlinear paraproduct $\Pi_{\ll}$ will allow us to modulate the parametric solution $\vartheta(\eta)$ with the quantity $a(u)=$ $\left(a_{1}(u), a_{2}(u)\right)$ in order to capture the most irregular part of the solution $u$ itself. As a consequence, the paracontrolled Ansatz

$$
u=\Pi_{\ll}(a(u), \vartheta)+u^{\sharp}
$$

will define a regular remainder term $u^{\sharp}$ which solves a standard PDE. With this decomposition the resonant products appearing in equation (4) can be estimated along the lines of the standard paracontrolled arguments introduced in Gubinelli, Imkeller and Perkowski (2015), and all the arguments introduced there can be extended in a straightforward manner to the quasilinear setting.

Recently Otto and Weber (2016) and Bailleul, Debussche and Hofmanová (2016) investigated quasilinear SPDEs in the context of pathwise methods and in a range of regularities compatible with the ones we will consider in this paper.

- Otto and Weber (2016) obtained a local well-posedness result for equations of the form

$$
\partial_{t} u(t, x)-a(u(t, x)) \partial_{x}^{2} u(t, x)=f(u(t, x)) \xi(t, x), \quad(t, x) \in \mathbb{T}^{2},
$$

where both space and time variables take values in a one-dimensional periodic domain and their noise can be white in time but coloured in space, essentially behaving like a distribution of parabolic regularity in $(-4 / 3,1)$. In order to do that they introduce a specific notion of modelled function and related estimates.

- Bailleul, Debussche and Hofmanová (2016) obtain local well-posedness for the generalised parabolic Anderson model equation

$$
\partial_{t} u(t, x)-a(u(t, x)) \Delta u(t, x)=g(u(t, x)) \xi(x), \quad t \geq 0, x \in \mathbb{T}^{2} .
$$

The authors obtain the same result as the one presented in Section 6 of our work, without the machinery of nonlinear paraproducts introduced here, but using only the basic tools of paracontrolled analysis and some clever transformations.

Let us comment a bit on relations of our results with those contained in these two papers.

- The parametric controlled Ansatz of Otto and Weber (2016) is the main source of inspiration for the present work. We think that it is a very deep and fundamental observation which is quite orthogonal to the development of an alternative theory for singular SPDEs which is the main aim of their work. Our paper 
shows that this idea survives outside their specific methodology, in particular that Safanov's approach to Schauder estimates is not necessary to handle quasilinear equations, and a relatively straightforward extension of the paracontrolled approach is sufficient to encompass in a compact manner the results on quasilinear equations contained in their paper.

- While the approach of Bailleul, Debussche and Hofmanová (2016) is more straightforward than ours, it has fundamental limitations. In particular, we remark that the generalisation of (7) to matrix-valued diffusion coefficients $\left(a_{i j}\right)_{i, j}$, namely to equations of the form

$$
\partial_{t} u(t, x)-a_{i j}(u(t, x)) \frac{\partial^{2}}{\partial x_{i} \partial x_{j}} u(t, x)=g(u(t, x)) \xi, \quad t \geq 0, x \in \mathbb{T}^{2},
$$

is out of reach of the techniques used in Bailleul, Debussche and Hofmanová (2016), while can be treated directly in our framework and by Otto and Weber's approach.

- Another interesting observation we make in the present work is that the parametric Ansatz can pervade without problems all the coefficients of the equation. In particular, we can allow very general noise terms whose law itself depends on the solutions of the equation. This is one of the main novelties of this paper, not present in other works on singular SPDEs. See equation (2) above and Section 7 below for a detailed explanation of this case.

- Let us mention that Otto and Weber's parametric Ansatz has predecessors in the theory of rough paths and in standard stochastic analysis. Nonlinear versions of rough paths have been considered by one of the authors in order to study the Korteweg-de Vries equation [Gubinelli (2012)]. Nonlinear Young integrals were used by one of the authors in joint work with Catellier [Catellier and Gubinelli (2016)] to study the the regularising properties of sample paths of stochastic processes. See also the related work of Hu and Lê (2017) on differential systems in Hölder media. Relevant to this discussion of nonlinear variants of rough paths is the work of Bailleul on rough flows [Bailleul (2015)] and their application to homogenisation [Bailleul and Catellier (2017)]. By looking at the composition $f(g(x))$ as the action of the distribution $\delta_{g(x)}$ on the function $f$, nonlinear constructions can be linearised at the price of working in infinite-dimensional spaces: this is the approach chosen by Kelly and Melbourne to avoid nonlinear generalisations of rough path theory in their study of homogenisation of a fast-slow system with random initial conditions [Kelly and Melbourne (2017)]. It is also worth mentioning Kunita's theory of semimartingale vector fields [Kunita (1984)], which occupies a place in stochastic analysis quite similar to that occupied by these recent developments in the landscape of rough paths/paracontrolled distributions theories. A nonlinear generalisation of the classic bilinear paraproducts already appeared in the notion of paracomposition introduced by Alinhac [Bony (1991)]. 
Usefulness of paraproducts in the analysis of nonlinear PDEs is by now well established; see, for example, the seminal paper [Meyer (1980)], the early review [Bony (1991)] and the recent books [Alinhac and Gérard (2007) and Bahouri, Chemin and Danchin (2011)]. Let us mention also the interesting paper [Hörmander (1990)] where paradifferential operators allow to bypass the Nash-Moser fix-point theorem in some applications where the loss of regularity prevents straightforward use of standard Banach fix-point theorem. The main observation in that paper is that, with the aid of paradifferential operators, it is possible to identify a "corrected" problem for which standard Banach fix point applies.

Paracontrolled calculus for singular SPDEs has been introduced by Gubinelli, Imkeller and Perkowski (2015) [see also the lecture notes on Gubinelli and Perkowski (2015)] and used to study various equations like the KPZ equation [Gubinelli and Perkowski (2017)], the dynamic $\Phi_{3}^{4}$ model [Catellier and Chouk (2013)] in $d=3$ and its global well-posedness [Mourrat and Weber (2017)], the spectrum of the continuous Anderson Hamiltonian in $d=2$ [Allez and Chouk (2015)]. By using heat-semigroup techniques paracontrolled calculus has been extended to the manifold context by Bailleul and Bernicot (2016a).

Paracontrolled calculus is currently limited to "first-order" computations. This limitation is also ubiquitous in the present work. Even if, in practice, this is not a big issue, and the calculus is still able to deal with a large class of problems, it makes the paracontrolled approach less appealing for a general theory of singular SPDEs. Let us remark that recently Bailleul and Bernicot (2016b) developed an higher order version of the paracontrolled calculus. However, apart from these recent developments, whose impact is still to be assessed, the most general theory for singular SPDEs has been developed in Friz and Hairer (2014), Hairer (2014a, 2014b) under the name of regularity structures theory. Regularity structures are a vast generalisation of Lyons' rough paths [Lyons (1998), Lyons, Caruana and Lévy (2007), Lyons and Qian (2002)] which give effective tools to describe nonlinear operations acting on certain spaces of distributions, their renormalisation by subtraction of local singularities and their use to solve singular SPDEs. Regularity structures have been successfully applied to all the models mentioned so far [Hairer $(2013,2014 a)$ ], to other models like the Sine-Gordon model [Hairer and Shen (2016)] (which however can also be handled via paracontrolled techniques) and to study weak universality conjectures [Hairer and Quastel (2015), Hairer and $\mathrm{Xu}$ (2016)]. In their current instantiation, it does not seem possible to solve quasilinear SPDEs via regularity structures.

The results of the present paper hint to the fact that a nonlinear version of regularity structures is conceivable, at least, in principle. Indeed one can imagine models depending on additional parameters and modelled distributions acting as evaluations of the parametric models at certain space-time dependent values of the parameters. During the revision stage of the present article, Hairer and Gerencsér uploaded a paper on the arXiv [Gerencsér and Hairer (2017)] in which they set up 
parameter-dependent regularity structures and the corresponding BPHZ models to solve a wide range of quasilinear stochastic PDEs (in particular those with noise regularity that is out of reach of standard first-order paracontrolled calculus).

The structure of the paper is the following. In Section 2, we introduce our basic tools: the nonlinear paraproduct decomposition and some related commutation lemmas. In Section 3, we introduce the paracontrolled Ansatz which allows us to transform the singular problem (1) into a well-behaved PDE. In Section 4, we discuss a priori estimates, the uniqueness of the solution of the transformed PDE and its continuity w.r.t. the random data and the initial condition. We also introduce the algebraic structure which allows us to renormalise the model. Section 5 deals with the renormalisation of the stochastic data and the construction of the enhanced noise associated to white noise. Section 6 deals with the extension of the results to the more general equation (7). In Section 7, we develop a paracontrolled structure for equation (2) and define its renormalised enhanced noise. Finally, in the Appendix, we review some reference material on Besov spaces and linear paraproducts, and prove some technical lemmas.

Notations. We will denote as $\mathscr{C}^{\alpha}:=B_{\infty, \infty}^{\alpha}\left(\mathbb{T}^{2}\right)$ the Zygmund space of regularity $\alpha \in \mathbb{R}$ on the torus $\mathbb{T}^{2}$. See the Appendix for the definition of the general Besov spaces $B_{p, q}^{\alpha}$, the Littlewoord-Paley operators $\left(\Delta_{i}\right)_{i \geq-1}$ and the basic properties thereof needed in this paper. If $V$ is a Banach space and $T>0$, we denote $C_{T}^{\alpha} V$ the space of $\alpha$-Hölder functions in $C_{T} V:=C([0, T] ; V)$. We introduce parabolic spaces $\mathscr{L}_{T}^{\alpha}:=C_{T}^{\alpha / 2} \mathscr{C}^{0} \cap C_{T} \mathscr{C}^{\alpha}$ with norm

$$
\|f\|_{\mathscr{L}_{T}^{\alpha}}=\|f\|_{C_{T}^{\alpha / 2} \mathscr{C}^{0}}+\|f\|_{C_{T} \mathscr{C}^{\alpha}} .
$$

Moreover, for convenience, we denote $\mathscr{C}_{T}^{\alpha}:=C_{T} \mathscr{C}^{\alpha}$. We will avoid writing explicitly the time span $T$ whenever this does not cause ambiguities. We will need also spaces for functions of $(\eta, t, x)$ where $\eta$ is an additional parameter in $[\lambda, 1]$ for $\lambda \in(0,1)$ which we denote $C_{\eta}^{k} V$ with the norm

$$
\|F\|_{C_{\eta}^{k} V}=\sup _{\eta \in[\lambda, 1]} \sup _{n=0, \ldots, k}\left\|\partial_{\eta}^{n} F(\eta, \cdot)\right\|_{V},
$$

where $V$ is a Banach space of functions on $[0, T] \times \mathbb{T}^{2}$ : in our case $V=\mathscr{C}_{T}^{\alpha}$ or $V=\mathscr{L}_{T}^{\alpha}$.

We will denote by $K_{i, x}(y)=2^{2 i} K\left(2^{i}(x-y)\right)$ the kernel of the LittlewoodPaley operator $\Delta_{i}$ and $Q_{i, t}(s)=Q_{i}(t-s)=2^{2 i} Q\left(2^{2 i}(t-s)\right)$ a smoothing kernel at scale $2^{2 i}$ in the time direction where $Q$ is a smooth, positive function with compact support in $\mathbb{R}_{+}$and mass 1 . We introduce also the shortcut $P_{i, x}=K_{<i-1, x}=$ $\sum_{j<i-1} K_{i, x}$. Another notation shortcut widely used in this article is to write $\int_{x, y}$ for integrals on $\mathbb{T}^{2}$ or $\mathbb{R}$ with respect to the measures $\mathrm{d} x$ and $\mathrm{d} y$ without specifying the integration bounds, whenever this does not create ambiguity. Finally, we will note $\delta f_{s y}^{t x}=f(t, x)-f(s, y)$ and $\delta_{\tau} f_{s y}^{t x}=f(s, y)+\tau(f(t, x)-f(s, y))$ for $\tau \in[0,1]$. 
2. Nonlinear paraproducts. In this section, we introduce nonlinear paraproduct and some related results that will be used in Section 3 to analyse equation (1).

Let $g:[0, T] \times \mathbb{T}^{2} \rightarrow \mathbb{R}$, and $h: \mathbb{R} \times[0, T] \times \mathbb{T}^{2} \rightarrow \mathbb{R}$ be smooth functions. We can decompose the composition $h(g(\cdot), \cdot)$ via nonlinear paraproducts as follows. Define

$$
\begin{aligned}
\Pi_{\prec}(g, h)(t, x) & :=\sum_{q \geq 1} \int_{y, z} P_{q, x}(y) K_{q, x}(z) h(g(t, y), t, z), \\
\Pi_{\circ}(g, h)(t, x) & :=\sum_{k \geq-1} \sum_{q=k-1}^{k+1} \int_{y, z} K_{k, x}(y) K_{q, x}(z) h(g(t, y), t, z), \\
\Pi_{\succ}(g, h)(t, x): & =\sum_{k \geq 1} \int_{y, z} K_{k, x}(z) P_{k, x}(y) h(g(t, z), t, y) .
\end{aligned}
$$

This gives a map

$$
\begin{aligned}
(g, h) & \mapsto \Pi_{\diamond}(g, h):=\Pi_{\prec}(g, h)+\Pi_{\circ}(g, h)+\Pi_{\succ}(g, h) \\
& =h(g(\cdot), \cdot)
\end{aligned}
$$

that can be uniquely extended to

$$
\Pi_{\diamond}: \mathscr{C}_{T}^{\rho} \times C_{\eta}^{2} \mathscr{C}_{T}^{\gamma} \rightarrow \mathscr{C}_{T}^{\gamma \wedge \rho}, \quad \rho \in(0,1), \gamma \in \mathbb{R}, \rho+\gamma>0,
$$

thanks to the following bounds:

LEMMA 2.1 (Nonlinear paraproduct estimates). Let $g \in \mathscr{C}_{T}^{\rho}$ for some $\rho \in$ $(0,1), g \in[\lambda, 1]$, and $h \in C_{\eta}^{2} \mathscr{C}_{T}^{\gamma}$ for any $\gamma \in \mathbb{R}$. Then

$$
\begin{aligned}
&\left\|\Pi_{\prec}(g, h)\right\|_{\mathscr{C}_{T}^{\gamma}} \lesssim\|h\|_{C_{\eta} \mathscr{C}_{T}^{\gamma}}, \\
&\left\|\Pi_{\succ}(g, h)\right\|_{\mathscr{C}_{T}^{\rho \wedge(\rho+\gamma)}} \lesssim\|g\|_{\mathscr{C}_{T}^{\rho}}\|h\|_{C_{\eta}^{1} \mathscr{C}_{T}^{\gamma}}
\end{aligned}
$$

and

$$
\begin{aligned}
& \left\|\Pi_{\prec}\left(g_{1}, h\right)-\Pi_{\prec}\left(g_{2}, h\right)\right\|_{\mathscr{C}_{T}^{\gamma}} \\
& \quad \lesssim\left\|g_{1}-g_{2}\right\|_{C_{T} L^{\infty}}\|h\|_{C_{\eta}^{1} \mathscr{C}_{T}^{\gamma}}, \\
& \left\|\Pi_{\succ}\left(g_{1}, h\right)-\Pi_{\succ}\left(g_{2}, h\right)\right\|_{\mathscr{C}_{T}^{\rho \wedge(\rho+\gamma)}} \\
& \quad \lesssim\left\|g_{1}-g_{2}\right\|_{C_{T} L^{\infty}}\left(\left\|g_{1}\right\|_{\mathscr{C}_{T}^{\rho}}+\left\|g_{2}\right\|_{\mathscr{C}_{T}^{\rho}}\right)\|h\|_{C_{\eta}^{2} \mathscr{C}_{T}^{\gamma}} \\
& \quad+\left\|g_{1}-g_{2}\right\|_{\mathscr{C}_{T}^{\rho}}\|h\|_{C_{\eta}^{1} \mathscr{C}_{T}^{\gamma}} .
\end{aligned}
$$


Moreover, if $\rho+\gamma>0$, we have also

$$
\begin{aligned}
& \left\|\Pi_{\circ}(g, h)\right\|_{\mathscr{C}_{T}^{\rho+\gamma}} \\
& \quad \lesssim\|h\|_{C_{\eta}^{1} \mathscr{C}_{T}^{\gamma}}\|g\|_{\mathscr{C}_{T}^{\rho}}, \\
& \left\|\Pi_{\circ}\left(g_{1}, h\right)-\Pi_{\circ}\left(g_{2}, h\right)\right\|_{\mathscr{C}_{T}^{\rho+\gamma}} \\
& \quad \lesssim\left\|g_{1}-g_{2}\right\|_{C_{T} L^{\infty}}\left(\left\|g_{1}\right\|_{\mathscr{C}_{T}^{\rho}}+\left\|g_{2}\right\|_{\mathscr{C}_{T}^{\rho}}\right)\|h\|_{C_{\eta}^{2} \mathscr{C}_{T}^{\gamma}} \\
& \quad+\left\|g_{1}-g_{2}\right\|_{\mathscr{C}_{T}^{\rho}}\|h\|_{C_{\eta}^{1} \mathscr{C}_{T}^{\gamma}} .
\end{aligned}
$$

In particular, if $\rho+\gamma>0$ the composition $\Pi_{\diamond}(g, h)=h(g(\cdot), \cdot)$ is linear in $h$ and locally Lipshitz in $g$ :

$$
\begin{aligned}
& \left\|\Pi_{\diamond}(g, h)\right\|_{\mathscr{C}_{T}^{\gamma}} \\
& \quad \therefore\|h\|_{C_{\eta}^{1} \mathscr{C}_{T}^{\gamma}}\|g\|_{\mathscr{C}_{T}^{\rho}}, \\
& \quad\left\|\Pi_{\diamond}\left(g_{1}, h\right)-\Pi_{\diamond}\left(g_{2}, h\right)\right\|_{\mathscr{C}_{T}^{\gamma}} \\
& \quad \lesssim\left\|g_{1}-g_{2}\right\|_{\mathscr{C}_{T}^{\rho}}\left(1+\left\|g_{1}\right\|_{\mathscr{C}_{T}^{\rho}}+\left\|g_{2}\right\|_{\mathscr{C}_{T}^{\rho}}\right)\|h\|_{C_{\eta}^{2} \mathscr{C} \gamma} .
\end{aligned}
$$

PROOF. Due to the support properties of the Fourier transforms of the kernels $K_{q}$ and $P_{q}$, it is easy to see that

$$
\int_{y, z} P_{q, x}(y) K_{q, x}(z) h(g(t, y), t, z)
$$

has Fourier transform compactly supported in an annulus $2^{q} \mathcal{A}$, and the same holds for

$$
\int_{y, z} K_{k, x}(z) P_{k, x}(y) h(g(t, z), t, y),
$$

while the resonant term

$$
\sum_{q=k-1}^{k+1} \int_{y, z} K_{k, x}(y) K_{q, x}(z) h(g(t, y), t, z)
$$

has Fourier transform supported in a ball of radius $2^{q}$. This allows us to estimate Besov norms of paraproducts via $L^{\infty}$ norms of Littlewood-Paley blocks in the usual way.

Using the fact that

$$
\begin{gathered}
\left\|\Delta_{k} h(g(t, y), t, \cdot)\right\|_{L^{\infty}} \lesssim 2^{-\gamma k}\|h\|_{C_{\eta} \mathscr{C}_{T}^{\nu}}, \\
\left\|\Delta_{k} h(g(t, y), t, \cdot)-\Delta_{k} h\left(g\left(t, y^{\prime}\right), t, \cdot\right)\right\|_{L^{\infty}} \lesssim 2^{-\gamma k}\|h\|_{C_{\eta}^{1} \mathscr{C}_{T}^{\gamma}}\|g\|_{\mathscr{C}_{T}^{\rho}}\left|y-y^{\prime}\right|^{\rho}
\end{gathered}
$$


and

$$
\left\|\Delta_{k} h\left(g_{1}(t, y), t, \cdot\right)-\Delta_{k} h\left(g_{2}(t, y), t, \cdot\right)\right\|_{L^{\infty}} \lesssim 2^{-\gamma k}\|h\|_{C_{\eta}^{1} \mathscr{C}_{T}^{\gamma}}\left\|g_{1}-g_{2}\right\|_{C_{T} L^{\infty}}
$$

we obtain the bounds on $\Pi_{\prec}(g, h), \Pi_{\succ}(g, h), \Pi_{\circ}(g, h), \Pi_{\prec}\left(g_{1}, h\right)-\Pi_{\prec}\left(g_{2}, h\right)$. We proceed to estimate the term $\Pi_{\succ}\left(g_{1}, h\right)-\Pi_{\succ}\left(g_{2}, h\right)$. We will use the following notation for brevity:

$$
\delta g_{2 z}^{1 y}:=g_{1}(t, y)-g_{2}(t, z) \quad \text { and } \quad \delta_{\tau} g_{2 z}^{1 y}:=g_{2}(t, z)+\tau\left(g_{1}(t, y)-g_{2}(t, z)\right) \text {. }
$$

Then

$$
\begin{aligned}
\mid \int_{y, z} & K_{k, x}(z) P_{k, x}(y)\left[h\left(g_{1}(t, z), t, y\right)-h\left(g_{2}(t, z), t, y\right)\right] \mid \\
= & \left|\int_{y, z, \tau \in[0,1]} K_{k, x}(z) P_{k, x}(y)\left[\partial_{\eta} h\left(\delta_{\tau} g_{2 z}^{1 z}, t, y\right) \delta g_{2 z}^{1 z}-\partial_{\eta} h\left(\delta_{\tau} g_{2 x}^{1 x}, t, y\right) \delta g_{2 x}^{1 x}\right]\right| \\
\lesssim & \left|\int_{y, z, t \in[0,1], \sigma \in[0,1]} K_{k, x}(z) P_{k, x}(y) \partial_{\eta}^{2} h\left(\delta_{\sigma}\left(\delta_{\tau} g_{2}^{1}\right)_{x}^{z}, t, y\right)\left(\delta g_{1 x}^{1 z}-\delta g_{2 x}^{2 z}\right) \delta g_{2 z}^{1 z}\right| \\
& +\left|\int_{y, z, \tau \in[0,1]} K_{k, x}(z) P_{k, x}(y) \partial_{\eta} h\left(\delta_{\tau} g_{2 x}^{1 x}, t, y\right)\left(\delta g_{2 z}^{1 z}-\delta g_{2 x}^{1 x}\right)\right| \\
\lesssim & \left\|g_{1}-g_{2}\right\|_{C_{T} L^{\infty}}\left(\left\|g_{1}\right\|_{C_{T} \mathscr{C} \rho}+\left\|g_{2}\right\|_{C_{T} \mathscr{C} \rho} \rho\|h\|_{C_{\eta}^{2} C_{T} \mathscr{C} \gamma} 2^{-\rho k} \sum_{q<k-1} 2^{-\gamma q}\right. \\
& +\left\|g_{1}-g_{2}\right\|_{C_{T} \mathscr{C} \rho}\|h\|_{C_{\eta}^{1} C_{T} \mathscr{C} \gamma} 2^{-\rho k} \sum_{q<k-1} 2^{-\gamma q} .
\end{aligned}
$$

With the same reasoning we can bound the norm of $\Pi_{\circ}\left(g_{1}, h\right)-\Pi_{\circ}\left(g_{2}, h\right)$.

We will need the following time-smoothed nonlinear paraproduct:

$$
\Pi_{\ll}(g, h)(t, x):=\sum_{i} \int_{y, s} Q_{i, t}(s) P_{i, x}(y)\left(\Delta_{i} h(g(s, y), t, \cdot)\right)(x),
$$

with $Q \in C_{c}^{\infty}(\mathbb{R})$ a kernel with total mass 1 as specified in the Introduction, and $Q_{i, t}(s):=2^{2 i} Q\left(2^{i}(t-s)\right)$. In (15), we use the convention that a continuous function $t \mapsto g(t)$ on $\mathbb{R}_{+}$is extended to $\mathbb{R}$ by defining $g(t)=g(0)$ for $t \leq 0$. This preserves the Hölder norms of index in $[0,1]$. The modified nonlinear paraproduct enjoys similar bounds to the regular one.

LeMmA 2.2. Let $g \in C_{T} L^{\infty}, g \in[\lambda, 1]$ and $h \in C_{\eta}^{1} \mathscr{L}_{T}^{\gamma}$ for $\gamma \in(0,2)$. Then

$$
\left\|\Pi_{\ll}(g, h)\right\|_{\mathscr{C}_{T}^{\gamma}} \lesssim\|h\|_{C_{\eta} \mathscr{C}_{T}^{\gamma}} \quad \text { and } \quad\left\|\Pi_{\ll}(g, h)\right\|_{\mathscr{L}_{T}^{\gamma}} \lesssim\|h\|_{C_{\eta} \mathscr{L}_{T}^{\gamma}} \text {. }
$$

Moreover, $\Pi_{\gtrless}(g, h)$ is linear in $h$ and

$$
\begin{gathered}
\left\|\Pi_{\ll}\left(g_{1}, h\right)-\Pi_{\ll}\left(g_{2}, h\right)\right\|_{\mathscr{C}_{T}^{\gamma}} \lesssim\left\|g_{1}-g_{2}\right\|_{C_{T} L} \infty\|h\|_{C_{\eta}^{1} \mathscr{C}_{T}^{\gamma}}, \\
\left\|\Pi_{\nVdash}\left(g_{1}, h\right)-\Pi_{\ll}\left(g_{2}, h\right)\right\|_{\mathscr{L}_{T}^{\gamma}} \lesssim\left\|g_{1}-g_{2}\right\|_{C_{T} L^{\infty}\|h\|_{C_{\eta}^{1} \mathscr{L}_{T}^{\gamma}} .}
\end{gathered}
$$


Proof. The norm $\left\|\Pi_{k}(g, h)\right\|_{C_{T} \mathscr{C} \gamma}$ can be treated in the same way as in Lemma 2.1.

We estimate $\left\|\Pi_{\ll}(g, h)\right\|_{C_{T}^{\gamma / 2} \mathscr{C}^{0}}$ as follows:

$$
\begin{aligned}
\left\|\Delta_{j} \Pi_{\ll}(g, h)\left(t_{1}\right)-\Delta_{j} \Pi_{\ll}(g, h)\left(t_{2}\right)\right\|_{L^{\infty}} \\
\quad \lesssim \sup _{x} \mid \int_{z} K_{j, x}(z) \sum_{i \sim j} \int_{y, s} Q_{i, t_{1}}(s) P_{i, z}(y)\left[\Delta_{i} h\left(g(s, y), t_{1}, z\right)\right. \\
\left.\quad-\Delta_{i} h\left(g(s, y), t_{2}, z\right)\right] \mid \\
\quad+\sup _{x}\left|\int_{z} K_{j, x}(z) \sum_{i \sim j} \int_{y, s}\left[Q_{i, t_{1}}(s)-Q_{i, t_{2}}(s)\right] P_{i, z}(y) \Delta_{i} h\left(g(s, y), t_{2}, z\right)\right| \\
\lesssim\left\|h\left(\cdot, t_{1}, \cdot\right)-h\left(\cdot, t_{2}, \cdot\right)\right\|_{C_{\eta} \mathscr{C}^{0}}+\left|t_{1}-t_{2}\right|^{\gamma / 2}\|h\|_{C_{\eta} \mathscr{C}_{T}^{\gamma}} .
\end{aligned}
$$

The second inequality can be obtained easily with the same techniques used so far.

REMARK 2.3. Using the Fourier support properties of the kernel $P_{q, x}(\cdot)$ it is easy to see that for every $x \in \mathbb{T}^{2}, q \geq 0: \int_{y} P_{q, x}(y)=\int_{y} K_{-1, x}(y)=1$ and $\int_{y} K_{q, x}(y)=0$. Then for a constant function $g(t, x)=\bar{g}$, one can write

$$
\Pi_{\diamond}(\bar{g}, h)=\Pi_{\prec}(\bar{g}, h)+\sum_{q \leq 0} \Delta_{q} h(\bar{g}, \cdot)
$$

and using the fact that the kernel $Q$ has mass 1 we have $\Pi_{\ll}(\bar{g}, h)=\Pi_{\prec}(\bar{g}, h)$.

2.1. Nonlinear commutator. The next technical ingredient is a commutator lemma between the nonlinear paraproduct of (15) and the standard resonant product. Since it will be needed below to analyse a term of the form $\Pi_{\ll}(g, h) \circ$ $\Delta \Pi_{\ll}(g, h)$, we will specialise our discussion to this specific structure. Notice that in the following the various space-time operators act pointwise in the parameter $\eta$, in the sense that, for example,

$$
(h \circ \Delta h)(\eta, t, x)=(h(\eta, t, \cdot) \circ \Delta h(\eta, t, \cdot))(x) .
$$

\section{Lemma 2.4. Define}

$$
\Lambda: C^{\infty}\left([0, T], \mathbb{T}^{2}\right) \times C_{\eta}^{2} C^{\infty}\left([0, T], \mathbb{T}^{2}\right) \rightarrow C^{\infty}\left([0, T], \mathbb{T}^{2}\right)
$$

by

$$
\Lambda(g, h):=\left[\Pi_{\ll}(g, h) \circ \Delta \Pi_{\ll}(g, h)\right]-\Pi_{\diamond}(g, h \circ \Delta h) .
$$


Then for all $\rho \in(0,1), \gamma<1 \varepsilon>0$ such that $2 \gamma-2+\rho-\varepsilon>0$ and $g \in[\lambda, 1]$, we have

$$
\begin{aligned}
& \|\Lambda(g, h)\|_{\mathscr{C}_{T}^{2 \gamma-2+\rho-\varepsilon}} \\
& \quad \lesssim\left(1+\|g\|_{\mathscr{L}_{T}^{\rho}}\right)\|h\|_{C_{\eta}^{1} \mathscr{C}_{T}^{\gamma}}^{2}, \\
& \left\|\Lambda\left(g_{1}, h\right)-\Lambda\left(g_{2}, h\right)\right\|_{\mathscr{C}_{T}^{2 \gamma-2+\rho-\varepsilon}} \\
& \quad \lesssim\left\|g_{1}-g_{2}\right\|_{C_{T} L^{\infty}}\left(\left\|g_{1}\right\|_{\mathscr{L}_{T}^{\rho}}+\left\|g_{2}\right\|_{\mathscr{L}_{T}^{\rho}}\right)\|h\|_{C_{\eta}^{2} \mathscr{C}_{T}^{\gamma}}^{2} \\
& \quad+\left\|g_{1}-g_{2}\right\|_{\mathscr{L}_{T}^{\rho}}\|h\|_{C_{\eta}^{1} \mathscr{C}_{T}^{\gamma}}^{2} .
\end{aligned}
$$

As a consequence, $\Lambda$ can be uniquely extended to a locally Lipshitz function

$$
\Lambda: \mathscr{L}_{T}^{\rho} \times C_{\eta}^{2} \mathscr{C}_{T}^{\gamma} \rightarrow \mathscr{C}_{T}^{2 \gamma-2+\rho-\varepsilon} .
$$

PROOF. We can approximate $\Pi_{\ll}(g, h) \circ \Delta \Pi_{\ll}(g, h)$ with its value for a fixed $g=g(t, z)$ to obtain

$$
\begin{aligned}
\Delta_{q} \Lambda(g, h)(t, x) & \\
= & \int_{z} K_{q, x}(z)\left(\Pi_{\ll}(g(t, z), h) \circ \Delta \Pi_{\ll}(g(t, z), h)\right)(t, z) \\
& -\int_{z} K_{q, x}(z) \Pi_{\diamond}(g, h \circ \Delta h)(t, z) \\
& +\int_{z} K_{q, x}(z)\left(\Pi_{\ll}(g, h) \circ \Delta\left(\Pi_{\ll}(g, h)-\Pi_{\ll}(g(t, z), h)\right)\right)(t, z) \\
& +\int_{z} K_{q, x}(z)\left(\left(\Pi_{\ll}(g, h)-\Pi_{\ll}(g(t, z), h)\right) \circ \Delta \Pi_{\ll}(g(t, z), h)\right)(t, z) .
\end{aligned}
$$

We start considering the first two terms in the expression above. Note that

$$
\Delta_{j} \Delta \Pi_{\ll}(g(t, z), h)(t, z)=\Delta_{j} \Pi_{\ll}(g(t, z), \Delta h)(t, z)
$$

and that by Remark $2.3 \forall i \geq 2$

$$
\Delta_{i} \Pi_{\ll}(g(t, z), h)(t, z)=\Delta_{i} h(g(t, z), t, \cdot)(z) .
$$

This yields

$$
\begin{gathered}
\left(\Pi_{\ll}(g(t, z), h) \circ \Delta \Pi_{\ll}(g(t, z), h)\right)(t, z)-\Pi_{\diamond}(g, h \circ \Delta h)(t, z) \\
=\sum_{i \sim j} \Delta_{i} \Pi_{\ll}(g(t, z), h)(t, z) \Delta_{j} \Pi_{\ll}(g(t, z), \Delta h)(t, z) \\
\quad-\sum_{i \sim j} \Delta_{i} h(g(t, z), t, \cdot)(z) \Delta_{j} \Delta h(g(t, z), t, \cdot)(z)
\end{gathered}
$$




$$
\begin{aligned}
= & \sum_{q=1}^{2} \Delta_{1} \Delta_{q} h(g(t, z), t, \cdot)(z) \sum_{\ell=1}^{2} \Delta_{1} \Delta_{\ell} \Delta h(g(t, z), t, \cdot)(z) \\
& -\sum_{i \sim j} \mathbb{1}_{i \leq 1} \mathbb{1}_{j \leq 1} \Delta_{i} h(g(t, z), t, \cdot)(z) \Delta_{j} \Delta h(g(t, z), t, \cdot)(z) .
\end{aligned}
$$

Note that fixing the value of the function $g$ in (18) makes it localised in Fourier space, that is, $\exists n_{0} \in \mathbb{N}$ such that $\forall k \geq n_{0}$ :

$$
\int_{z} K_{k, x}(z) \sum_{q=1}^{2} \Delta_{1} \Delta_{q} h(g(t, x), t, \cdot)(z) \sum_{\ell=1}^{2} \Delta_{1} \Delta_{\ell} \Delta h(g(t, x), t, \cdot)(z)=0
$$

and this last term can be easily bound in $L^{\infty}$ by $\|h\|_{C_{\eta} \mathscr{C}_{T}^{\gamma}}^{2}$. Thus, we add and subtract to (18) the terms

$$
\begin{aligned}
& \sum_{q=1}^{2} \Delta_{1} \Delta_{q} h(g(t, x), t, \cdot)(z) \sum_{\ell=1}^{2} \Delta_{1} \Delta_{\ell} \Delta h(g(t, x), t, \cdot)(z), \\
& \sum_{q=1}^{2} \Delta_{1} \Delta_{q} h(g(t, z), t, \cdot)(z) \sum_{\ell=1}^{2} \Delta_{1} \Delta_{\ell} \Delta h(g(t, x), t, \cdot)(z)
\end{aligned}
$$

and we are left estimating

$$
\begin{aligned}
\int_{z} K_{k, x}(z) & \sum_{q=1}^{2} \Delta_{1} \Delta_{q} h(g(t, z), t, \cdot)(z) \\
& \cdot \sum_{\ell=1}^{2} \Delta_{1}\left[\Delta_{\ell} \Delta h(g(t, z), t, \cdot)-\Delta_{\ell} \Delta h(g(t, x), t, \cdot)\right](z)
\end{aligned}
$$

and

$$
\begin{gathered}
\int_{z} K_{k, x}(z) \sum_{q=1}^{2} \Delta_{1}\left[\Delta_{q} h(g(t, z), t, \cdot)-\Delta_{q} h(g(t, x), t, \cdot)\right](z) \\
\cdot \sum_{\ell=1}^{2} \Delta_{1} \Delta_{\ell} \Delta h(g(t, x), t, \cdot)(z) .
\end{gathered}
$$

As already noted in the proof of Lemma 2.1, we have

$$
\begin{aligned}
& \left\|\Delta_{q} h(g(t, z), t, \cdot)-\Delta_{k} h(g(t, x), t, \cdot)\right\|_{L^{\infty}} \\
& \lesssim 2^{-\gamma q}\|h\|_{C_{\eta}^{1} \mathscr{C}_{T}^{\gamma}}\|g\|_{\mathscr{C}_{T}^{\rho}}|z-x|^{\rho}, \\
& \left\|\Delta_{\ell} \Delta h(g(t, z), t, \cdot)-\Delta_{\ell} \Delta h(g(t, x), t, \cdot)\right\|_{L^{\infty}} \\
& \lesssim 2^{(2-\gamma) \ell}\|h\|_{C_{\eta}^{1} \mathscr{C}_{T}^{\gamma}}\|g\|_{\mathscr{C}_{T}^{\rho}}|z-x|^{\rho}
\end{aligned}
$$


and this, together with the estimation

$$
\int_{z}\left|K_{k, x}(z)\right||z-x|^{\rho} \lesssim 2^{-k \rho}
$$

allows us to bound the terms above in $L^{\infty}$ with $2^{-k \rho}\|g\|_{\mathscr{C}_{T}^{\rho}}\|h\|_{C_{\eta}^{1} \mathscr{C}_{T}^{\gamma}}^{2}$. Summing up, we have seen that (18) can be bound in $\mathscr{C}_{T}^{\rho}$ by $\left(1+\|g\|_{\mathscr{C}_{T}^{\rho}}\right)\|h\|_{C_{\eta}^{1} \mathscr{C}_{T}^{\gamma}}^{2}$, and this gives a bound on $\mathscr{C}_{T}^{2 \gamma-2+\rho-\varepsilon}$ for $\gamma<1, \varepsilon>0$. The exact same reasoning can be applied to (19) to obtain the same estimation.

Consider now (16) and (17). We obtain

$$
\begin{gathered}
\int_{z} K_{q, x}(z)\left[\left(\Pi_{\ll}(g, h)-\Pi_{\ll}(g(t, z), h)\right) \circ \Delta \Pi_{\ll}(g(t, z), h)\right](t, z) \\
=\int_{z} K_{q, x}(z) \sum_{i \sim j \gtrsim q} \Delta_{j} \Delta \Pi_{\ll}(g(t, z), h)(t, z) \\
\cdot\left(\Delta_{i} \Pi_{\ll}(g, h)(t, z)-\Delta_{i} \Pi_{\ll}(g(t, z), h)(t, z)\right) .
\end{gathered}
$$

Using Lemma 2.2 we have

$$
\left|\Delta \Delta_{j} \Pi_{\ll}(g(t, z), h)(t, z)\right| \lesssim 2^{(2-\gamma) j}\|h\|_{C_{\eta} \mathscr{C}_{T}^{\gamma}} .
$$

Lemma 2.5 gives

$$
\left|\Delta_{i}\left(\Pi_{\ll}(g, h)-\Pi_{\ll}(g(t, z), h)\right)(t, z)\right| \lesssim 2^{-(\gamma+\rho-\varepsilon) i}\|g\|_{\mathscr{L}_{T}^{\rho}}\|h\|_{C_{\eta}^{1} \mathscr{C}_{T}^{\gamma}},
$$

and thus (17) is bounded by $2^{-(2 \gamma+\rho-2-\varepsilon) q}\|g\|_{\mathscr{L}_{T}^{\rho}}\|h\|_{\mathscr{C}_{T}^{\gamma}}^{2}$. We can easily bound (16) in the same way, and this proves the first inequality.

The second result of this lemma can be obtained following the same reasoning as above, noting that $\forall k, q \geq-1$ :

$$
\begin{aligned}
\int_{z} K_{k, x}(z)\left[\Delta_{q} h\left(g_{1}(t, z), t, \cdot\right)(z)-\Delta_{q} h\left(g_{1}(t, x), t, \cdot\right)(z)\right. \\
\left.+\Delta_{q} h\left(g_{2}(t, x), t, \cdot\right)(z)-\Delta_{q} h\left(g_{2}(t, z), t, \cdot\right)(z)\right] \\
\quad \lesssim 2^{-\rho k}\left\|g_{1}-g_{2}\right\|_{\mathscr{C}_{T}^{\rho}}\|h\|_{C_{\eta}^{1} \mathscr{C}_{T}^{\gamma}} \\
\quad+2^{-\rho k}\left\|g_{1}-g_{2}\right\|_{C_{T} L^{\infty}}\left(\left\|g_{1}\right\|_{\mathscr{C}_{T}^{\rho}}+\left\|g_{2}\right\|_{\mathscr{C}_{T}^{\rho}}\right)\|h\|_{C_{\eta}^{2} \mathscr{C}_{T}^{\gamma}}
\end{aligned}
$$

and using the estimations of Lemma 2.5.

The extension of $\Lambda$ to $\mathscr{L}_{T}^{\rho} \times C_{\eta}^{2} \mathscr{L}_{T}^{\gamma}$ is standard [see, e.g., the proof of the commutator lemma, Gubinelli, Imkeller and Perkowski (2015), Lemma 2.4].

\section{LEMMA 2.5. Let us introduce the shortcut notation}

$$
\wp_{i}(g, h)(t, z):=\Delta_{i} \Pi_{\ll}(g, h)(t, z)-\Delta_{i} \Pi_{\ll}(g(t, z), h)(t, z) .
$$


Then, with the same assumptions of Lemma 2.4, we have

$$
\left|\wp_{i}(g, h)(t, z)\right| \lesssim 2^{(\varepsilon-\rho-\gamma) i}\|g\|_{\mathscr{L}_{T}^{\rho}}\|h\|_{C_{\eta}^{1} \mathscr{C}_{T}^{\gamma}}
$$

and

$$
\begin{aligned}
& \left|\wp_{i}\left(g_{1}, h\right)(t, z)-\wp_{i}\left(g_{2}, h\right)(t, z)\right| \\
& \qquad 2^{(\varepsilon-\rho-\gamma) i}\left\|g_{1}-g_{2}\right\|_{\mathscr{L}_{T}^{\rho}}\|h\|_{C_{\eta}^{1} \mathscr{C}_{T}^{\gamma}} \\
& \quad+2^{(\varepsilon-\rho-\gamma) i}\left\|g_{1}-g_{2}\right\|_{C_{T} L^{\infty}}\left(\left\|g_{1}\right\|_{\mathscr{L}_{T}^{\rho}}+\left\|g_{2}\right\|_{\mathscr{L}_{T}^{\rho}}\right)\|h\|_{C_{\eta}^{2} \mathscr{C}_{T}^{\gamma}} .
\end{aligned}
$$

PROOF.

$$
\begin{aligned}
{\left[\Delta_{i} \Pi_{\ll}\right.} & \left.(g, h)-\Delta_{i} \Pi_{\ll}(g(t, z), h)\right](t, z) \\
= & \sum_{k \sim i} \int_{\substack{x, y \\
s, \tau}} K_{i, z}(x) Q_{k, t}(s) P_{k-1, x}(y) \partial_{\eta} \Delta_{k} h\left(\delta_{\tau} g_{t z}^{s y}, t, x\right)\left(\delta g_{t y}^{s y}+\delta g_{t z}^{t y}\right) \\
\lesssim & \sum_{k \sim i} \int_{\substack{x, y \\
s, \tau}}\left|K_{i, z}(x) Q_{k, t}(s) P_{k-1, x}(y)\right| \\
& \cdot\left\|\partial_{\eta} \Delta_{k} h\right\|_{C_{T} L^{\infty}|t-s|^{(\rho-\varepsilon) / 2}\|g\|_{C_{T}^{\rho / 2-\varepsilon / 2} L^{\infty}}} \\
& \quad+\sum_{k \sim i} \int_{\substack{x, y \\
s, \tau}}\left|K_{i, z}(x) Q_{k, t}(s) P_{k-1, x}(y)\right|\left\|\partial_{\eta} \Delta_{k} h\right\|_{C_{T} L^{\infty}|y-z|^{\rho}\|g\|_{\mathscr{C}_{T}^{\rho}}} \\
\lesssim & 2^{-(\rho-\varepsilon) i} 2^{-\gamma i}\left\|\partial_{\eta} h\right\|_{\mathscr{C}_{T}^{\gamma}}\left(\|g\|_{C_{T}^{\rho} \mathscr{C}^{0}}+\|g\|_{\mathscr{C}_{T}^{\rho}}\right),
\end{aligned}
$$

where we used the notation $\delta g_{t z}^{s y}=g(s, y)-g(t, z), \delta_{\tau} g_{t z}^{s y}=g(t, z)+\tau(g(s, y)-$ $g(t, z))$ and Lemma A.5. This proves the first bound.

The second inequality can be obtained in the same way with the techniques already used here and in Lemma 2.1.

2.2. Approximate paradifferential problem. In this section, we construct an approximate solution to the equation

$$
\left(\partial_{t}-g \prec \Delta\right) u=f, \quad u(0, \cdot)=0,
$$

with data $f \in \mathscr{C}^{\gamma-2}$ and $g \in \mathscr{L}_{T}^{\rho}$, for some fixed $\rho, \gamma \in(0,1)$. The idea is to obtain it via a certain class of paradifferential operators. We introduce the operator $\mathscr{L}$ acting on functions of $(\eta, t, x)$ by

$$
(\mathscr{L} U)(\eta, t, x):=\partial_{t} U(\eta, t, x)-\eta \Delta U(\eta, t, x) .
$$

We will also use the notation $\mathscr{L}_{\eta_{1}}:=\partial_{t}-\eta_{1} \Delta$. 
Observe that if $u$ does not depend on $\eta$ we can define

$$
\Pi_{\prec}(g, \mathscr{L}) u:=\Pi_{\prec}(g, \mathscr{L} u)
$$

and from Definition (11) with $h=\mathscr{L} u$ we obtain $\Pi_{\prec}(g, \mathscr{L}) u=\partial_{t} u-g \prec \Delta u$.

We can describe the commutation between the differential operator $\mathscr{L}$ and the paraproduct $\Pi_{\nVdash}(g, \cdot)$ via the following estimate.

LEMMA 2.6. Let $\rho \in(0,1), \gamma \in \mathbb{R}$. Let $U \in C_{\eta}^{2} \mathscr{C}_{T}^{\gamma}$ and $g \in \mathscr{L}_{T}^{\rho}$ such that $g \in[\lambda, 1]$. Define

$$
\Psi(g, U):=R_{1}+R_{2}
$$

with $R_{1}$ and $R_{2}$ as in (25), (26). Then for every $\varepsilon>0$,

$$
\|\Psi(g, U)\|_{\mathscr{C}_{T}^{\rho+\gamma-2-\varepsilon}} \lesssim\left(1+\|g\|_{C_{T} L^{\infty}}\right)\|g\|_{\mathscr{L}_{T}^{\rho}}\|U\|_{C_{\eta}^{1} \mathscr{C}_{T}^{\gamma}} .
$$

Moreover, $\Psi(g, U)$ is linear in $U$ and

$$
\begin{aligned}
& \left\|\Psi\left(g_{1}, U\right)-\Psi\left(g_{2}, U\right)\right\|_{\mathscr{C}_{T}^{\rho+\gamma-2-\varepsilon}} \\
& \quad \lesssim\left\|g_{1}-g_{2}\right\|_{\mathscr{L}_{T}^{\rho}}\left(1+\left\|g_{1}\right\|_{\mathscr{L}_{T}^{\rho}}+\left\|g_{2}\right\|_{\mathscr{L}_{T}^{\rho}}\right)\|U\|_{C_{\eta}^{2} \mathscr{C}_{T}^{\gamma}} .
\end{aligned}
$$

In particular, we have

$$
\Psi(g, U)=\Pi_{\ll}(g, \mathscr{L} U)-\Pi_{\prec}(g, \mathscr{L}) \Pi_{\ll}(g, U) \in C_{T} \mathscr{C}^{\rho+\gamma-2-\varepsilon}
$$

whenever this expression makes sense.

ProOF. We start considering $g \in C^{\infty}\left([0, T], \mathbb{T}^{2}\right)$ and $U \in C_{\eta}^{2} C^{\infty}\left([0, T], \mathbb{T}^{2}\right)$, and prove (24) in this setting. Notice that $\Pi_{\nVdash}\left(g(t, y), \mathscr{L}_{g(t, y)} U\right)=\mathscr{L} U(g(t$, $y))$. As a consequence, we can estimate

$$
\begin{aligned}
& \Pi_{\ll}(g, \mathscr{L} U)(t, x)-\Pi_{\prec}\left(g, \mathscr{L} \Pi_{\ll}(g, U)\right)(t, x) \\
& =\Pi_{\nVdash}(g, \mathscr{L} U)(t, x)-\sum_{k} \int_{y} P_{k, x}(y)\left(\mathscr{L}_{g(t, y)} \Delta_{k} \Pi_{\ll}(g, U)\right)(t, x) \\
& =\Pi_{\ll}(g, \mathscr{L} U)(t, x)-\sum_{k} \int_{y} P_{k, x}(y)\left(\partial_{t} \Delta_{k} \Pi_{\ll}(g, U)\right)(t, x) \\
& +\sum_{k} \int_{y} P_{k, x}(y) g(t, y)\left(\Delta \Delta_{k} \Pi_{\ll}(g, U)\right)(t, x) \\
& =\Pi_{\ll}\left(g, \mathscr{L} U-\partial_{t} U\right)(t, x) \\
& +\sum_{k} \int_{y} P_{k, x}(y) g(t, y)\left(\Delta_{k} \Pi_{\ll}(g, \Delta U)\right)(t, x) \\
& +\sum_{k} \int_{y} P_{k, x}(y) g(t, y)\left(\Delta_{k}\left[\Delta, \Pi_{\ll}(g, \cdot)\right] U\right)(t, x)
\end{aligned}
$$




$$
-\sum_{k} \int_{y} P_{k, x}(y)\left(\Delta_{k}\left[\partial_{t}, \Pi_{k}(g, \cdot)\right] U\right)(t, x)
$$

with the commutators

$$
\begin{aligned}
& {\left[\Delta, \Pi_{\ll}(g, \cdot)\right] U:=\Delta \Pi_{\ll}(g, U)-\Pi_{\ll}(g, \Delta U),} \\
& {\left[\partial_{t}, \Pi_{\ll}(g, \cdot)\right] U:=\partial_{t} \Delta_{k} \Pi_{\ll}(g, U)-\Delta_{k} \Pi_{\ll}\left(g, \partial_{t} U\right) .}
\end{aligned}
$$

We have

$$
\begin{aligned}
\Pi_{\ll}\left(g, \mathscr{L} U-\partial_{t} U\right)(t, x)+\sum_{k} \int_{y} P_{k, x}(y) g(t, y)\left(\Delta_{k} \Pi_{\ll}(g, \Delta U)\right)(t, x) \\
\quad=R_{1}(t, x)
\end{aligned}
$$

with the definition

$$
\begin{aligned}
R_{1}(t, x):= & \sum_{k, i} \int_{y_{y, z}^{\prime}, s} P_{k, x}(y) K_{k, x}(z) P_{i, z}\left(y^{\prime}\right) Q_{i, t}(s)[g(t, y) \\
& \left.-g\left(s, y^{\prime}\right)\right] \Delta \Delta_{i} U\left(g\left(s, y^{\prime}\right), t, z\right)
\end{aligned}
$$

and

$$
\begin{aligned}
& \sum_{k} \int_{y} P_{k, x}(y)\left[g(t, y)\left(\Delta_{k}\left[\Delta, \Pi_{\ll}(g, \cdot)\right] U\right)(t, x)\right. \\
& \left.-\left(\Delta_{k}\left[\partial_{t}, \Pi_{\ll}(g, \cdot)\right] U\right)(t, x)\right] \\
& \quad=R_{2}(t, x)
\end{aligned}
$$

with the definition

$$
\begin{aligned}
R_{2}(t, x):= & \sum_{k, i} \int_{y, y^{\prime}, s} P_{k, x}(y) K_{k, x}(z) Q_{i, t}(s) g(t, y) \Delta P_{i, z}\left(y^{\prime}\right) \Delta_{i} U\left(g\left(s, y^{\prime}\right), t, z\right) \\
& +2 \sum_{k, i} \int_{y, y^{\prime}, s} P_{k, x}(y) K_{k, x}(z) Q_{i, t}(s) g(t, y) \\
& \times \nabla P_{i, z}\left(y^{\prime}\right) \nabla \Delta_{i} U\left(g\left(s, y^{\prime}\right), t, z\right) \\
& -\sum_{k, i} \int_{y, y^{\prime}, s} P_{k, x}(y) K_{k, x}(z) \partial_{t} Q_{i, t}(s) P_{i, z}\left(y^{\prime}\right) \Delta_{i} U\left(g\left(s, y^{\prime}\right), t, z\right) .
\end{aligned}
$$


Indeed,

$$
\begin{aligned}
& \left(\left[\partial_{t}, \Pi_{\ll}(g, \cdot)\right] U\right)(t, x) \\
& =\sum_{i} \int_{y, s}\left(\partial_{t} Q_{i, t}\right)(s) P_{i, x}(y)\left(\Delta_{i} U(g(s, y), t, x)\right), \\
& \left(\left[\Delta, \Pi_{\nVdash}(g, \cdot)\right] U\right)(t, x) \\
& =\sum_{i} \int_{y, s} Q_{i, t}(s) \Delta P_{i, x}(y)\left(\Delta_{i} U(g(s, y), t, x)\right) \\
& \quad+2 \sum_{i} \int_{y, s} Q_{i, t}(s) \nabla P_{i, x}(y)\left(\nabla \Delta_{i} U(g(s, y), t, x)\right) .
\end{aligned}
$$

This shows that (24) holds for smooth functions.

With the techniques used in Lemma 2.5, we can estimate

$$
\left|\Delta_{q} R_{1}(t, x)\right| \lesssim \sum_{k \sim q}\left(2^{-(\rho-\varepsilon) k}\|g\|_{C_{T}^{\rho / 2} \mathscr{C}^{0}}+2^{-\rho k}\|g\|_{\mathscr{C}_{T}^{\rho}}\right) 2^{(2-\gamma) k}\|U\|_{C_{\eta} \mathscr{C}_{T}^{\gamma}} .
$$

By the spectral support properties of the commutators, we have that

$$
\left\|\left[\Delta, \Pi_{\ll}(g, \cdot)\right] U\right\|_{\mathscr{C}_{T}^{\gamma+\rho-2}} \lesssim\|g\|_{\mathscr{C}_{T}^{\rho}}\|U\|_{C_{\eta}^{1} \mathscr{C}_{T}^{\gamma}}
$$

and

$$
\begin{aligned}
\left\|\Delta_{q}\left[\partial_{t}, \Pi_{\ll}(g, \cdot)\right] U\right\|_{C_{T} L^{\infty}} \lesssim & 2^{(2+\varepsilon-\rho-\gamma) q}\|g\|_{C_{T}^{\rho / 2} \mathscr{C} 0}\|U\|_{C_{\eta}^{1} \mathscr{C}_{T}^{\gamma}} \\
& +2^{(2-\rho-\gamma) q}\|g\|_{\mathscr{C}_{T}^{\rho}}\|U\|_{C_{\eta}^{1} \mathscr{C}_{T}^{\gamma}} .
\end{aligned}
$$

This yields

$$
\left\|R_{2}\right\|_{\mathscr{C}_{T}^{\gamma+\rho-2-\varepsilon}} \lesssim\left(1+\|g\|_{C_{T} L^{\infty}}\right)\|g\|_{\mathscr{L}_{T}^{\rho}}\|U\|_{C_{\eta}^{1} \mathscr{C}_{T}^{\gamma}} .
$$

We have so far proved (23) and then (24) follows by continuity. The local Lipshitz dependence on $g$ can be obtained via similar computations.

REMARK 2.7. If $f$ does not depend on $\eta$ we consider the parametric problem,

$$
\left(\partial_{t}-\eta \Delta\right) U_{f}(\eta, t)=f, \quad U_{f}(\eta, 0)=0, \quad \eta \in[\lambda, 1],
$$

which is solved by

$$
U_{f}(\eta, t)=\int_{0}^{t} e^{\eta \Delta(t-s)} f \mathrm{~d} s
$$

Remark that

$$
\begin{aligned}
& \partial_{\eta} U_{f}(\eta, t)=\int_{0}^{t} e^{\eta \Delta(t-s)}(t-s) \Delta f \mathrm{~d} s \quad \text { and } \\
& \partial_{\eta}^{2} U_{f}(\eta, t)=\int_{0}^{t} e^{\eta \Delta(t-s)}(t-s)^{2} \Delta^{2} f \mathrm{~d} s .
\end{aligned}
$$


We have, thanks to the well-known Schauder estimates of Lemma A.4 (since $\eta \geq$ $\lambda)$ :

$$
\left\|U_{f}\right\|_{C_{\eta}^{2} \mathscr{L}_{T}^{\gamma}}:=\sup _{n=0,1,2} \sup _{\eta \in[\lambda, 1]}\left\|\partial_{\eta}^{n} U_{f}(\eta)\right\|_{\mathscr{L}_{T}^{\gamma}} \lesssim\|f\|_{\mathscr{C}_{T}^{\gamma-2}}
$$

We define then

$$
u(t, x):=\Pi_{\ll}\left(g, U_{f}\right)(t, x)
$$

and observe that $u(t, x)$ is an approximate solution of equation (20), indeed

$$
\begin{aligned}
\left(\partial_{t}-g \prec \Delta\right) u & =\Pi_{\prec}\left(g, \mathscr{L} \Pi_{\gtrless}\left(g, U_{f}\right)\right)=\Pi_{\ll}\left(g, \mathscr{L} U_{f}\right)-\Psi\left(g, U_{f}\right) \\
& =f-\Psi\left(g, U_{f}\right)
\end{aligned}
$$

and the estimation in Lemma 2.6 together with the bound (29) yield immediately the following inequality:

$$
\left\|\Psi\left(g, U_{f}\right)\right\|_{\mathscr{C}_{T}^{\rho+\gamma-2-\varepsilon}} \lesssim\|g\|_{\mathscr{L}_{T}^{\rho}}\left(1+\|g\|_{C_{T} L^{\infty}}\right)\|f\|_{\mathscr{C}_{T}^{\gamma-2}}
$$

3. Paracontrolled Ansatz. In order to give a meaning to the PDE in (4) with initial condition $u_{0} \in \mathscr{C}^{\alpha}$, our initial goal will be to get information on solutions $\theta=\theta(g)$ of the equation

$$
\partial_{t} \theta-g \prec \Delta \theta=\xi,
$$

for a fixed $g \in \mathscr{C}_{T}^{\alpha}, 2 / 3<\alpha<1, g \in[\lambda, 1]$. Using the results of Section 2.2, we consider to this effect the parametric problem

$$
\left(\partial_{t}-\eta \Delta\right) \vartheta(\eta, t)=\xi
$$

for $\eta \in[\lambda, 1]$. We will consider the stationary solution of this problem which has the form

$$
\vartheta(\eta, x)=\int_{0}^{\infty} e^{\eta \Delta s} \xi \mathrm{d} s=(-\eta \Delta)^{-1} \xi
$$

and in order for (32) to be well defined we impose that the noise $\xi$ has zero mean on $\mathbb{T}^{2}$ (this is a simplifying assumption which can be easily removed, e.g., at the price of adding a linear term to the equation). We can control (32) by bounding its Littlewood-Paley blocks with a Bernstein lemma for distributions with compactly supported Fourier transform [Bahouri, Chemin and Danchin (2011), Lemma 2.1] to obtain

$$
\|\vartheta\|_{C_{\eta}^{2} \mathscr{L}_{T}^{\alpha}}=\|\vartheta\|_{C_{\eta}^{2} \mathscr{C}_{T}^{\alpha}} \lesssim\|\xi\|_{\mathscr{C}^{\alpha-2}} .
$$

We define now for every $t \in[0, T]$

$$
\theta(t, x):=\Pi_{\nVdash}(a(u), \vartheta) .
$$


Thanks to Lemma 2.2, we have the bound $\|\theta\|_{\mathscr{L}_{T}^{\alpha}} \lesssim\|\vartheta\|_{C_{\eta} \mathscr{L}_{T}^{\alpha}} \lesssim\|\xi\|_{\mathscr{C}^{\alpha-2}}$. We observe that this definition together with Lemma 2.6 gives

$$
\partial_{t} \theta-a(u) \prec \Delta \theta=\xi-\Psi(a(u), \vartheta)
$$

with $\|\Psi(a(u), \vartheta)\|_{\mathscr{C}_{T}^{2 \alpha-2-\varepsilon}} \lesssim\|a(u)\|_{\mathscr{L}_{T}^{\alpha}}^{2}\|\xi\|_{\mathscr{C}_{T}^{\alpha-2}}$. We expect then $\Psi(a(u), \vartheta)$ to be bounded in $\mathscr{C}_{T}^{2 \alpha-2-\varepsilon}$ for any $\varepsilon>0$. At this point let us introduce the Ansatz

$$
u=\theta+u^{\#} \text {. }
$$

REMARK 3.1. Notice that we are not making any assumption on the existence of such $u$, which is the subject of Section 4. Our aim here is to find the equation that a couple $\left(u, u^{\sharp}\right) \in \mathscr{C}_{T}^{\alpha} \times \mathscr{C}_{T}^{2 \alpha}$ verifying (34) must solve, in order for $u$ to solve (4).

Observe that

$$
\begin{aligned}
\partial_{t} u-a(u) \prec \Delta u & =\left(\partial_{t}-a(u) \prec \Delta\right) \theta+\left(\partial_{t}-a(u) \prec \Delta\right) u^{\sharp} \\
& =\xi+\left(\partial_{t}-a(u) \prec \Delta\right) u^{\sharp}-\Psi(a(u), \vartheta) .
\end{aligned}
$$

It follows that $u^{\sharp}$ must solve

$$
\left\{\begin{array}{l}
\left(\partial_{t}-a(u) \prec \Delta\right) u^{\sharp}=\Phi(u)+\Psi(a(u), \vartheta), \\
u^{\sharp}(t=0)=u_{0}^{\sharp}:=u_{0}-\Pi_{\ll}\left(a\left(u_{0}\right), \vartheta\right)(t=0) \in \mathscr{C}^{\alpha}
\end{array}\right.
$$

with $\Phi(u)=a(u) \circ \Delta u+a(u) \succ \Delta u$, and if we can make sense of the resonant term $a(u) \circ \Delta u$, it is reasonable to expect $u^{\sharp}(t, \cdot) \in \mathscr{C}^{2 \alpha} \forall t \in(0, T]$. Indeed, take $U^{\sharp}:=U_{Q}$ to be the solution of

$$
\mathscr{L} U^{\sharp}(\eta):=\left(\partial_{t}-\eta \Delta\right) U^{\sharp}(\eta)=Q, \quad U^{\sharp}(\eta, t=0)=0
$$

for some $Q=Q\left(u^{\sharp}\right)$ to be determined and $\eta \in[\lambda, 1]$. Using again Lemma 2.6 as shown in Remark 2.7 we have

$$
\left(\partial_{t}-a(u) \prec \Delta\right) \Pi_{\ll}\left(a(u), U^{\sharp}\right)=Q\left(u^{\sharp}\right)-\Psi\left(a(u), U^{\sharp}\right) .
$$

For $\eta \in[\lambda, 1]$, we define $\mathscr{P}_{t} u_{0}^{\sharp}(\eta):=e^{\eta \Delta t} u_{0}^{\sharp}$ so that $\mathscr{L}\left(\mathscr{P}_{t} u_{0}^{\sharp}\right)=0$, with $\mathscr{L}$ as in $(21)$.

We set

$$
u^{\sharp}:=\Pi_{\ll}\left(a(u), U^{\sharp}\right)+\Pi_{\ll}\left(a(u), \mathscr{P} u_{0}^{\sharp}\right) .
$$

Taking

$$
Q\left(u^{\sharp}\right):=\Phi(u)+\Psi(a(u), \vartheta)+\Psi\left(a(u), U^{\sharp}\right)+\Psi\left(a(u), \mathscr{P} u_{0}^{\sharp}\right),
$$

we obtain that $U^{\sharp}$ solves equation (36) if and only if $u^{\sharp}$ solves equation (35). As we will see, $Q\left(u^{\sharp}\right)(t)$ belongs to $\mathscr{C}^{2 \alpha-2} \forall t \in(0, T]$ but not uniformly as $t \rightarrow 0$. However, it belongs to $\mathscr{C}^{\alpha-2}$ uniformly as $t \rightarrow 0$. 
It remains to control the resonant term $a(u) \circ \Delta u$ appearing in $\Phi(u)$. We have

$$
a(u) \circ \Delta u=a(u) \circ \Delta \theta+a(u) \circ \Delta u^{\sharp} .
$$

By paralinearisation (see Theorem A.8) $a(u)=a^{\prime}(u) \prec u+R_{a}(u)$ with $\left\|R_{a}(u)\right\|_{\mathscr{C}_{T}^{2 \alpha}} \lesssim 1+\|u\|_{\mathscr{C}_{T}^{\alpha}}^{2}$, and then

$$
a(u) \circ \Delta \theta=\left(a^{\prime}(u) \prec u\right) \circ \Delta \theta+R_{a}(u) \circ \Delta \theta .
$$

In order to use the commutator lemma (Lemma A.9), we can estimate $a^{\prime}(u)$, recalling that $\alpha \in(0,1)$, as

$$
\left\|a^{\prime}(u)\right\|_{\mathscr{C}_{T}^{\alpha}} \lesssim\left\|a^{\prime \prime}\right\|_{L^{\infty}}\|u\|_{\mathscr{C}_{T}^{\alpha}}
$$

and write

$$
a(u) \circ \Delta \theta=a^{\prime}(u)(u \circ \Delta \theta)+C\left(a^{\prime}(u), u, \Delta \theta\right)+R_{a}(u) \circ \Delta \theta .
$$

Then Ansatz (34) gives

$$
a(u) \circ \Delta \theta=a^{\prime}(u)(\theta \circ \Delta \theta)+a^{\prime}(u)\left(u^{\sharp} \circ \Delta \theta\right)+C\left(a^{\prime}(u), u, \Delta \theta\right)+R_{a}(u) \circ \Delta \theta \text {. }
$$

Summarising, we have

$$
\begin{aligned}
\Phi(u)= & a^{\prime}(u)(\theta \circ \Delta \theta)+a(u) \succ \Delta u+a^{\prime}(u)\left(u^{\sharp} \circ \Delta \theta\right) \\
& +C\left(a^{\prime}(u), u, \Delta \theta\right)+R_{a}(u) \circ \Delta \theta+a(u) \circ \Delta u^{\sharp} .
\end{aligned}
$$

Thanks to the nonlinear commutator (Lemma 2.4), we can decompose the resonant term $\theta \circ \Delta \theta$ to obtain

$$
\begin{aligned}
\Phi(u)= & a(u) \succ \Delta u+a^{\prime}(u)\left(u^{\sharp} \circ \Delta \theta\right)+C\left(a^{\prime}(u), u, \Delta \theta\right)+R_{a}(u) \circ \Delta \theta \\
& +a^{\prime}(u) \Lambda(a(u), \vartheta)+a^{\prime}(u) \Pi_{\diamond}\left(a(u), \Theta_{2}\right)+a(u) \circ \Delta u^{\sharp}
\end{aligned}
$$

and $\Lambda(a(u), \vartheta) \in C_{T} L^{\infty}$ if $u \in \mathscr{L}_{T}^{\alpha}$. Here, we defined

$$
\Theta_{2}(\eta, x):=(\vartheta \circ \Delta \vartheta)(\eta, x)=\sum_{i \sim j} \Delta_{i} \vartheta(\eta, \cdot)(x) \Delta_{j}[\Delta \vartheta(\eta, \cdot)](x) .
$$

Finally, recalling the decomposition of $u^{\sharp}$ in two terms (37) we obtain

$$
\Phi(u)=a^{\prime}(u) \Pi_{\diamond}\left(a(u), \Theta_{2}\right)+\Phi_{1}(u)+\Phi_{2}(u),
$$

where

$$
\begin{aligned}
\Phi_{1}(u):= & a(u) \succ \Delta u+C\left(a^{\prime}(u), u, \Delta \theta\right)+R_{a}(u) \circ \Delta \theta+a^{\prime}(u) \Lambda(a(u), \vartheta) \\
& +a^{\prime}(u)\left(\Pi_{\ll}\left(a(u), U^{\sharp}\right) \circ \Delta \theta\right)+a(u) \circ \Delta \Pi_{\ll}\left(a(u), U^{\sharp}\right), \\
\Phi_{2}(u):= & a^{\prime}(u)\left(\Pi_{\ll}\left(a(u), \mathscr{P} u_{0}^{\sharp}\right) \circ \Delta \theta\right)+a(u) \circ \Delta \Pi_{\ll}\left(a(u), \mathscr{P} u_{0}^{\sharp}\right) .
\end{aligned}
$$


Thanks to Lemma 2.1 the terms $a^{\prime}(u) \Pi_{\diamond}\left(a(u), \Theta_{2}\right)$ and $\Phi_{1}(u)$ can be estimated in $\mathscr{C}_{T}^{2 \alpha-2}$, provided $\Theta_{2} \in C_{\eta}^{2} \mathscr{C}_{T}^{2 \alpha-2}$ (see Section 5). On the other hand, the term $\Phi_{2}(u)(t)$ can be estimated in $\mathscr{C}^{2 \alpha-2}$ only for strictly positive times $t>0$ due to the lack of regularity of the initial condition $u_{0}^{\sharp}$ which a priori lives only in $\mathscr{C}^{\alpha}$.

Note moreover that the specific form of $\Phi$ allows us to deduce that if we replace $\Theta_{2}$ by $\tilde{\Theta}_{2}=\Theta_{2}-H$ with $H \in C_{\eta}^{2} \mathscr{C}_{T}^{2 \alpha-2}$ then this is equivalent to consider an equation for $u$ of the form

$$
\partial_{t} u(t, x)-a(u(t, x)) \Delta u(t, x)=\xi(x)-a^{\prime}(u(t, x)) H(a(u(t, x)), t, x) .
$$

Let us resume this long discussion in the following theorem.

TheOREM 3.2. Assume that $\xi \in \mathscr{C}^{0}, u_{0} \in \mathscr{C}^{2}, H \in C_{\eta}^{2} \mathscr{C}_{T}^{0} . u \in C_{T}^{1} \mathscr{C}^{2}$ is the classical solution to the equation

$$
\begin{aligned}
\partial_{t} u(t, x)-a(u(t, x)) \Delta u(t, x) & =\xi(x)-a^{\prime}(u(t, x)) H(a(u(t, x)), t, x), \\
u(0) & =u_{0},
\end{aligned}
$$

up to time $T>0$ if

$$
u=\Pi_{\ll}\left(a(u), \vartheta+U^{\sharp}+\mathscr{P} u_{0}^{\sharp}\right),
$$

where $\vartheta$ is the solution to equation (32) and $U^{\sharp}$ is the solution to the PDE

$$
\left(\partial_{t}-\eta \Delta\right) U^{\sharp}(\eta)=F\left(u, U^{\sharp}, u_{0}^{\sharp}\right), \quad U^{\sharp}(\eta, 0)=0, \quad \eta \in[\lambda, 1]
$$

with

$$
\begin{aligned}
F\left(u, U^{\sharp}, u_{0}\right)= & a^{\prime}(u) \Pi_{\diamond}\left(a(u), \Theta_{2}\right)+\Phi_{1}(u)+\Phi_{2}(u)+\Psi(a(u), \vartheta) \\
& +\Psi\left(a(u), U^{\sharp}\right)+\Psi\left(a(u), \mathscr{P} u_{0}^{\sharp}\right)
\end{aligned}
$$

and $\Theta_{2}=\vartheta \circ \Delta \vartheta-H$.

Definition 3.3. For any $\alpha \in \mathbb{R}$ we define $\mathcal{X}^{\alpha} \subseteq C_{\eta}^{2} \mathscr{C}^{\alpha} \times C_{\eta}^{2} \mathscr{C}^{2 \alpha-2}$ the closure of the image of the map

$$
(\rho, H) \in C_{\eta}^{2} \mathscr{C}^{2} \times C_{\eta}^{2} \mathscr{C}^{0} \quad \mapsto \quad J(\rho, H)=(\rho, \rho \circ \Delta \rho-H) \in C_{\eta}^{2} \mathscr{C}^{2} \times C_{\eta}^{2} \mathscr{C}^{0}
$$

(in the topology of $C_{\eta}^{2} \mathscr{C}^{\alpha} \times C_{\eta}^{2} \mathscr{C}^{2 \alpha-2}$ ).

We call the elements in $\mathcal{X}^{\alpha}$ enhanced noises. In the next section, we will exploit the space $\mathcal{X}^{\alpha}$ for $2 / 3<\alpha<1$ to solve equations (40) and (34). 
4. Local well-posedness. The main result of this section is the local wellposedness for equations (34) and (40) when $\left(\vartheta, \Theta_{2}\right) \in \mathcal{X}^{\alpha}$ and $u_{0} \in \mathscr{C}^{\alpha}$ for $2 / 3<$ $\alpha<1$. This yields a unique solution to (39), thanks to Theorem 3.2.

THEOREM 4.1. Let $\alpha \in\left(\frac{2}{3}, 1\right)$. Then for any $\left(\vartheta, \Theta_{2}\right) \in \mathcal{X}^{\alpha}$ and $u_{0} \in \mathscr{C}^{\alpha}$ there exists a time $T>0$ depending only on $\left\|\left(\vartheta, \Theta_{2}\right)\right\| \mathcal{X}^{\alpha}$ and $\left\|u_{0}\right\|_{\alpha}$ up to which the system of equations (34) and (40) has a unique solution $\left(u, U^{\sharp}\right) \in \mathscr{L}_{T}^{\alpha} \times C_{\eta}^{2} \mathscr{L}_{T}^{2 \delta}$ for all $\delta<\alpha$ such that $2 \delta+\alpha>2$. For any fixed $\tau>0$, there exist a ball $B_{\tau} \subseteq$ $\mathscr{C}^{\alpha} \times \mathcal{X}^{\alpha}$ such that the solution map

$$
\Sigma_{\tau}:\left(u_{0}, \vartheta, \Theta_{2}\right) \in B_{\tau} \quad \mapsto \quad\left(u, U^{\sharp}\right) \in \mathscr{L}_{\tau}^{\alpha} \times C_{\eta}^{2} \mathscr{L}_{\tau}^{2 \delta}
$$

is well defined and Lipshitz continuous in the data.

REMARK 4.2. The proof is based on a Picard fixed-point argument. In order to have a contraction map on a small time interval $[0, T]$, we carry on our analysis of $U^{\sharp}$ in the space $C_{\eta}^{2} \mathscr{L}_{T}^{2 \delta} \supset C_{\eta}^{2} \mathscr{L}_{T}^{2 \alpha}$ and make use of the estimates of Lemma A.4 to obtain a factor $T^{\varepsilon}$ for some (small) $\varepsilon>0$.

THEOREM 4.1. Let $\mathcal{G}_{T}=\mathscr{L}_{T}^{\alpha} \times C_{\eta}^{2} \mathscr{L}_{T}^{2 \delta}$. We introduce the map

$$
\Gamma:\left(u, U^{\sharp}\right) \in \mathcal{G}_{T} \quad \mapsto \quad\left(\Gamma_{u}\left(u, U^{\sharp}\right), \Gamma_{U^{\sharp}}\left(u, U^{\sharp}\right)\right) \in \mathcal{G}_{T}
$$

by

$$
\Gamma_{u}\left(u, U^{\sharp}\right):=\Pi_{\ll}(a(u), \vartheta)+\Pi_{\ll}\left(a(u), \Gamma_{U^{\sharp}}\left(u, U^{\sharp}\right)\right)+\Pi_{\ll}\left(a(u), \mathscr{P} u_{0}^{\sharp}\right)
$$

and

$$
\left(\partial_{t}-\eta \Delta\right) \Gamma_{U^{\sharp}}\left(u, U^{\sharp}\right)(\eta)=F\left(u, U^{\sharp}, u_{0}^{\sharp}\right)
$$

with $\Gamma_{U^{\sharp}}\left(u, U^{\sharp}\right)(\eta)(0)=0, \eta \in[\lambda, 1]$. We will establish that this map is a contraction in the space $\mathcal{G}_{T}$.

First we have to show that there exists a ball $B \subset \mathcal{G}_{T}$ such that $\Gamma(B) \subseteq B$. We have the bound $\left\|\mathscr{P} u_{0}^{\sharp}\right\|_{C_{\eta}^{1} \mathscr{L}_{T}^{\alpha}} \lesssim\left\|u_{0}^{\sharp}\right\|_{\mathscr{C}_{T}^{\alpha}}$. It is easy to obtain, using the estimates of Section 2 and Lemma A.4:

$$
\begin{aligned}
& \left\|\int_{0}^{T} e^{-\eta \Delta(t-s)}\left[\Phi_{1}(u)+\Psi\left(a(u), \vartheta+U^{\sharp}+\mathscr{P} u_{0}^{\sharp}\right)\right]_{s} \mathrm{~d} s\right\|_{C_{\eta}^{2} \mathscr{L}_{T}^{2 \delta}} \\
& \quad \lesssim T^{\kappa}\left(1+\|u\|_{\mathscr{L}_{T}^{\alpha}}\right)^{4}\left(1+\|\xi\|_{\mathscr{C}^{\alpha-2}}\right)^{2}\left\|u_{0}^{\sharp}\right\|_{\mathscr{C}^{\alpha}}\left(1+\left\|U^{\sharp}\right\|_{C_{\eta}^{2} \mathscr{L}_{T}^{2 \delta}}\right)
\end{aligned}
$$

for some $\kappa>0$.

By the assumption that $\left(\vartheta, \Theta_{2}\right) \in \mathcal{X}^{\alpha}$, we deduce that there exists $M>0$ such that $\left\|\Theta_{2}\right\|_{C_{\eta}^{2} \mathscr{C}_{T}^{2 \alpha-2}} \leq M$. We have

$$
\begin{gathered}
\left\|\int_{0}^{T} e^{-\eta \Delta(t-s)}\left[a^{\prime}(u) \Pi_{\diamond}\left(a(u), \Theta_{2}\right)\right]_{S} \mathrm{~d} s\right\|_{C_{\eta}^{2} \mathscr{L}_{T}^{2 \delta}} \\
\lesssim T^{\alpha-\delta}\left(1+\|u\|_{\mathscr{C}_{T}^{\alpha}}\right)^{2}\left\|\Theta_{2}\right\|_{C_{\eta}^{2} \mathscr{C}_{T}^{2 \alpha-2} .}
\end{gathered}
$$


To bound the term $\Phi_{2}(u)$, we observe that $\left\|\mathscr{P}_{t} u_{0}^{\sharp}\right\|_{C_{\eta}^{2} \mathscr{C}^{2 \alpha}} \lesssim t^{-\frac{\alpha}{2}}\left\|u_{0}^{\sharp}\right\|_{\mathscr{C}}$ a thanks to Lemma A.4. This gives

$$
\left\|\int_{0}^{T} e^{-\eta \Delta(t-s)} \Phi_{2}(u)_{s} \mathrm{~d} s\right\|_{C_{\eta}^{2} \mathscr{L}_{T}^{2 \delta}} \lesssim T^{\alpha-\delta}\left(1+\|u\|_{\mathscr{C}_{T}^{\alpha}}\right)\left(1+\|\xi\|_{\mathscr{C}^{\alpha}-2}\right)\left\|u_{0}^{\sharp}\right\|_{\mathscr{C}^{\alpha}}
$$

and then $\Gamma_{U^{\sharp}}\left(u, U^{\sharp}\right)$ is bounded in $C_{\eta}^{2} \mathscr{L}_{T}^{2 \delta}$ for $T$ small enough. We have also

$$
\begin{aligned}
\left\|\Gamma_{u}\left(u, U^{\sharp}\right)\right\|_{\mathscr{L}_{T}^{\alpha}} & \lesssim\|\xi\|_{\mathscr{C}^{\alpha-2}}+\left\|u_{0}^{\sharp}\right\|_{\mathscr{C}^{\alpha}}+\left\|\Gamma_{U^{\sharp}}\left(u, U^{\sharp}\right)\right\|_{C_{\eta} \mathscr{L}_{T}^{\alpha}} \\
& \lesssim\|\xi\|_{\mathscr{C}^{\alpha-2}}+\left\|u_{0}^{\sharp}\right\|_{\mathscr{C}^{\alpha}}+T^{\frac{2 \delta-\alpha}{2}}\left\|\Gamma_{U^{\sharp}}\left(u, U^{\sharp}\right)\right\|_{C_{\eta}^{2} \mathscr{L}_{T}^{2 \delta}}
\end{aligned}
$$

and these bounds show that $\Gamma(B) \subseteq B$. The contractivity of $\Gamma_{U^{\sharp}}\left(u, U^{\sharp}\right)$ can be obtained in the same way. Now consider $\Gamma_{u}\left(u, U^{\sharp}\right)$ : we have

$$
\begin{aligned}
& \left\|\Pi_{\ll}\left(a\left(u_{1}\right), U_{1}^{\sharp}\right)-\Pi_{\ll}\left(a\left(u_{2}\right), U_{2}^{\sharp}\right)\right\|_{\mathscr{L}_{T}^{\alpha}} \\
& \quad \lesssim T^{\frac{2 \delta-\alpha}{2}}\left(\left\|U_{1}^{\sharp}-U_{2}^{\sharp}\right\|_{C_{\eta} \mathscr{L}^{2 \delta}}+\left\|u_{1}-u_{2}\right\|_{C_{T} L^{\infty}}\left\|U_{2}^{\sharp}\right\|_{C_{\eta}^{1} \mathscr{L}^{2 \delta}}\right)
\end{aligned}
$$

while for the other terms in $\Gamma_{u}\left(u_{1}, U_{1}^{\sharp}\right)-\Gamma_{u}\left(u_{2}, U_{2}^{\sharp}\right)$ we remark that

$$
\sup _{s \in[0, t]}\left\|u_{1, s}-u_{0}-u_{2, s}+u_{0}\right\|_{L^{\infty}} \lesssim t^{\varepsilon / 2}\left\|u_{1}-u_{2}\right\|_{C_{[0, t]}^{\varepsilon / 2} L^{\infty}}
$$

Then $\forall 0<\varepsilon<\alpha$, using Lemma 2.2 and Lemma A.5:

$$
\begin{aligned}
\left\|\Pi_{\nVdash}\left(a\left(u_{1}\right), \vartheta\right)-\Pi_{\ll}\left(a\left(u_{2}\right), \vartheta\right)\right\|_{\mathscr{L}_{T}^{\alpha}} & \lesssim\left\|a\left(u_{1}\right)-a\left(u_{2}\right)\right\|_{C_{T} L^{\infty}}\|\vartheta\|_{C_{\eta}^{1} \mathscr{L}_{T}^{\alpha}} \\
& \lesssim\left\|u_{1}-u_{2}\right\| C_{T} L^{\infty}\|\xi\|_{\mathscr{C}^{\alpha-2}} \\
& \lesssim T^{\varepsilon / 2}\left\|u_{1}-u_{2}\right\|_{C_{T}^{\varepsilon / 2} L^{\infty}}\|\xi\|_{\mathscr{C}^{\alpha-2}} \\
& \lesssim T^{\varepsilon / 2}\left\|u_{1}-u_{2}\right\|_{\mathscr{L}_{T}^{\alpha}}\|\xi\|_{\mathscr{C}^{\alpha-2}} .
\end{aligned}
$$

With the same reasoning, we estimate

$$
\begin{aligned}
\| \Pi_{\ll} & \left(a\left(u_{1}\right), \mathscr{P} u_{0}^{\sharp}\right)-\Pi_{\ll}\left(a\left(u_{2}\right), \mathscr{P} u_{0}^{\sharp}\right) \|_{\mathscr{L}_{T}^{\alpha}} \\
& \lesssim T^{\varepsilon / 2}\left\|u_{1}-u_{2}\right\|_{C_{T}^{\varepsilon / 2} L^{\infty}}\left\|\mathscr{P} u_{0}^{\sharp}\right\|_{C_{\eta}^{1} \mathscr{L}_{T}^{\alpha}} \\
& \lesssim T^{\varepsilon / 2}\left\|u_{1}-u_{2}\right\|_{\mathscr{L}_{T}^{\alpha}}\left\|u_{0}^{\sharp}\right\|_{\mathscr{C}_{T}^{\alpha}}
\end{aligned}
$$

and then $\Gamma$ is a contraction for small times.

The uniqueness of the solution $\left(u, U^{\sharp}\right) \in \mathscr{L}_{T}^{\alpha} \times C_{\eta}^{2} \mathscr{L}_{T}^{2 \delta}$ and the Lipshitz continuity of the localised solution map $\Sigma_{\tau}$ can be proved along the same lines via standard arguments. 
5. Renormalisation. At this point, we want to construct an enhanced noise $\Xi$ associated to the white noise $\xi$. Already in the standard setting of the generalised PAM model with constant diffusion matrix, the construction of the enhancement requires a renormalisation since the resonant product $\vartheta \circ \Delta \vartheta$ is not well defined.

Let $\psi \in \mathcal{S}\left(\mathbb{T}^{2}\right)$ be a cutoff function and let $\psi_{\varepsilon}(x)=\varepsilon^{-2} \psi(x / \varepsilon)$. Then define a regularised noise by $\xi_{\varepsilon}=\psi_{\varepsilon} * \xi$ and let $\vartheta_{\varepsilon}=(-\eta \Delta)^{-1} \xi_{\varepsilon}$. Notice that

$$
\begin{aligned}
H_{\varepsilon}(\eta) & :=\mathbb{E}\left[\vartheta_{\varepsilon}(\eta, x) \circ \Delta \vartheta_{\varepsilon}(\eta, x)\right]=\mathbb{E}\left[\vartheta_{\varepsilon}(\eta, x) \Delta \vartheta_{\varepsilon}(\eta, x)\right] \\
& =-\sum_{k \in \mathbb{Z}^{2} \backslash\{0\}} \frac{\hat{\psi}_{\varepsilon}(k)^{2}}{\eta^{2}|k|^{2}}=-\frac{\sigma_{\varepsilon}}{\eta^{2}},
\end{aligned}
$$

where

$$
\sigma_{\varepsilon}:=\sum_{k \in \mathbb{Z}^{2} \backslash\{0\}} \frac{\hat{\psi}_{\varepsilon}(k)^{2}}{|k|^{2}} \simeq|\log \varepsilon|
$$

as $\varepsilon \rightarrow 0$. Subtracting the diverging quantity $H_{\varepsilon}$ to $\vartheta_{\varepsilon} \circ \Delta \vartheta_{\varepsilon}$ and then taking the limit as $\varepsilon \rightarrow 0$ delivers a finite result.

THEOREM 5.1. Take $\alpha<1$ and let $\Xi_{\varepsilon}=\left(\xi_{\varepsilon}, \Xi_{2, \varepsilon}\right):=\left(\xi_{\varepsilon}, \vartheta_{\varepsilon} \circ \Delta \vartheta_{\varepsilon}-H_{\varepsilon}\right)$. Then the family $\left(\Xi_{\varepsilon}\right)_{\varepsilon} \subseteq \mathcal{X}^{\alpha}$ converges a.s. and in $L^{p}$ to a random element $\Xi=$ $\left(\xi, \Xi_{2}\right) \in \mathcal{X}^{\alpha}$.

PROOF. The proof is a mild modification of the proof for PAM [Gubinelli, Imkeller and Perkowski (2015)]. In order to establish the required $C_{\eta}^{2} \mathscr{C}_{T}^{2 \alpha-2}$ regularity for $\Xi_{2}$, we follow the computations for the case where the diffusion coefficient is constant. We only have to discuss the additional regularity in the parameter $\eta$. In order to do so, observe that

$$
\Xi_{2, \varepsilon}(\eta)=\sum_{i \sim j} \llbracket \Delta_{i} \vartheta_{\varepsilon}(\eta) \Delta_{j} \Delta \vartheta_{\varepsilon}(\eta) \rrbracket,
$$

where $\llbracket \cdot \rrbracket$ denotes the Wick product with respect to the Gaussian structure of $\xi$. Then we have

$$
\partial_{\eta} \Xi_{2, \varepsilon}(\eta)=\sum_{i \sim j} \llbracket \Delta_{i} \partial_{\eta} \vartheta_{\varepsilon}(\eta) \Delta_{j} \Delta \vartheta_{\varepsilon}(\eta) \rrbracket+\sum_{i \sim j} \llbracket \Delta_{i} \vartheta_{\varepsilon}(\eta) \Delta_{j} \Delta \partial_{\eta} \vartheta_{\varepsilon}(\eta) \rrbracket,
$$

and

$$
\begin{aligned}
\partial_{\eta}^{2} \Xi_{2, \varepsilon}(\eta)= & \sum_{i \sim j} \llbracket \Delta_{i} \partial_{\eta}^{2} \vartheta_{\varepsilon}(\eta) \Delta_{j} \Delta \vartheta_{\varepsilon}(\eta) \rrbracket+\sum_{i \sim j} \llbracket \Delta_{i} \vartheta_{\varepsilon}(\eta) \Delta_{j} \Delta \partial_{\eta}^{2} \vartheta_{\varepsilon}(\eta) \rrbracket \\
& +\sum_{i \sim j} 2 \llbracket \Delta_{i} \partial_{\eta} \vartheta_{\varepsilon}(\eta) \Delta_{j} \Delta \partial_{\eta} \vartheta_{\varepsilon}(\eta) \rrbracket .
\end{aligned}
$$


Now the computations relative to the regularities of these additional stochastic objects are equivalent to those for the term $\Xi_{2, \varepsilon}$ where one or two instances of $\vartheta_{\varepsilon}(\eta)$ are replaced by Gaussian fields of similar regularities of the form $\partial_{\eta} \vartheta_{\varepsilon}(\eta)$ and $\partial_{\eta}^{2} \vartheta_{\varepsilon}(\eta)$. A direct inspection of the proof contained in Gubinelli, Imkeller and Perkowski (2015) allows us to deduce that we have almost sure $\mathscr{C}^{2 \alpha-2}$ regularity for these terms and also for random fields $\partial_{\eta}^{n} \Xi_{2, \varepsilon}$ for any finite $n$. This allows us also to deduce that the random field is almost surely smooth in the parameter $\eta$. Similar computations allow to prove continuity in $\varepsilon$ for $\varepsilon>0$. The rest of the proof is standard.

In conclusion, we see that in order to be able to use this convergence result we need to modify our approximate PDE and consider instead

$$
\partial_{t} u_{\varepsilon}-a\left(u_{\varepsilon}\right) \Delta u_{\varepsilon}=\xi_{\varepsilon}-a^{\prime}\left(u_{\varepsilon}\right) H_{\varepsilon}\left(a\left(u_{\varepsilon}\right)\right)
$$

which gives the renormalised equation (3).

Our well-posedness results for the paracontrolled formulation of this equation together with the convergence result of Theorem 5.1 allow to deduce that $u_{\varepsilon} \rightarrow u$ in $\mathscr{C}_{T}^{\delta}$ for any $2 / 3<\delta<\alpha<1$ and that the limiting process $u$ satisfies a modified version of eq. (1), namely

$$
\partial_{t} u-a(u) \diamond \Delta u=\xi, \quad u(0)=u_{0},
$$

where $a(u) \diamond \Delta u$ denotes a renormalised diffusion term given by

$$
a(u) \diamond \Delta u:=a(u) \prec \Delta u+a^{\prime}(u) \Pi_{\diamond}\left(a(u), \Xi_{2}\right)+\Phi_{1}(u)+\Phi_{2}(u) .
$$

6. Nonlinear source terms. Let us start by discussing the presence of a $u$ dependent r.h.s. in equation (1). We want to solve

$$
\partial_{t} u-a_{1}(u) \Delta u=a_{2}(u) \xi,
$$

where $a_{1}$ is a nonlinear diffusion coefficient as before and $a_{2}: \mathbb{R} \rightarrow \mathbb{R}$ is another bounded function with sufficiently many bounded derivatives. We rewrite this equation as

$\Pi_{\prec}(a(u), \mathscr{L}) u=a_{2}(u) \prec \xi+a_{1}(u) \circ \Delta u+a_{2}(u) \circ \xi+a_{1}(u) \succ \Delta u+a_{2}(u) \succ \xi$, where now $a(u)=\left(a_{1}(u), a_{2}(u)\right)$ is a vector valued nonlinearity. Since we do not need $u$ to depend on any parameter $\eta=\left(\eta_{1}, \eta_{2}\right)$, we have defined $\mathscr{L}$ as

$$
\mathscr{L}(\eta):=\partial_{t}-\eta_{1} \Delta
$$

and used the identity $\Pi_{\prec}(a(u), \mathscr{L}) u=\left(\partial_{t}-a_{1}(u) \prec \Delta\right) u$, similar to what we have done in (22).

Notice that the nonlinear paraproduct can be extended trivially to the vector valued case in such a way that, for example,

$$
\Pi_{\ll}\left(\left(g_{1}, g_{2}\right), h\right)(t, x)=\sum_{i} \int_{y, s} Q_{i, t}(s) P_{i, x}(y)\left(\Delta_{i} h\left(\left(g_{1}(s, y), g_{2}(s, y)\right), t, \cdot\right)\right)(x) \text {. }
$$


As before, we make the Ansatz

$$
u=\Pi_{\ll}(a(u), \vartheta)+u^{\sharp},
$$

where now $\vartheta$ solves

$$
\mathscr{L}(\eta) \vartheta(\eta)=\left(\partial_{t}-\eta_{1} \Delta\right) \vartheta(\eta)=\eta_{2} \xi,
$$

for $\eta=\left(\eta_{1}, \eta_{2}\right) \in[\lambda, 1] \times[-L, L]$ where $L$ is a large but fixed constant. The bounded domain is important to be able to have uniform estimates and reuse the estimates proved above in the simple situation of $\eta_{2}=1$. The solution of this equation is

$$
\vartheta(\eta, \cdot)=\eta_{2} \int_{0}^{\infty} e^{\eta_{1} \Delta s} \xi \mathrm{d} s=-\frac{\eta_{2}}{\eta_{1}} \Delta^{-1} \xi .
$$

Observe that

$$
\Pi_{\prec}(a(u), \mathscr{L}) u=\Pi_{\prec}(a(u), \mathscr{L}) \Pi_{\ll}(a(u), \vartheta)+\Pi_{\prec}(a(u), \mathscr{L}) u^{\sharp}
$$

and recall that by Lemma 2.6

$$
\Pi_{\prec}(a(u), \mathscr{L}) \Pi_{\ll}(a(u), \vartheta)=\Pi_{\ll}(a(u), \mathscr{L} \vartheta)+\Psi(a(u), \vartheta) .
$$

Now

$$
(\mathscr{L} \vartheta)(\eta)=\left(\partial_{t}-\eta_{1} \Delta\right) \vartheta(\eta, t, x)=\Xi(\eta), \quad \eta=\left(\eta_{1}, \eta_{2}\right) \in[\lambda, 1] \times[-L, L]
$$

with $\Xi(\eta)(t, x)=\eta_{2} \xi(x)$ and then

$$
\Pi_{\ll}(a(u), \mathscr{L} \vartheta)=\Pi_{\ll}(a(u), \Xi)=a_{2}(u) \ll \xi .
$$

In conclusion,

$$
\Pi_{\prec}(a(u), \mathscr{L}) \Pi_{\ll}(a(u), \vartheta)=a_{2}(u) \ll \xi+\Psi(a(u), \vartheta)
$$

and the equation for $u^{\sharp}$ reads

$$
\begin{aligned}
\Pi_{\prec}\left(a(u), \mathscr{L} u^{\sharp}\right)= & a_{1}(u) \circ \Delta u+a_{2}(u) \circ \xi+\left[a_{2}(u) \prec \xi-a_{2}(u) \prec \xi\right] \\
& +a_{1}(u) \succ \Delta u+a_{2}(u) \succ \xi-\Psi(a(u), \vartheta),
\end{aligned}
$$

where now all the terms on the r.h.s. can be considered remainder terms. Let us just remark that the commutation term $a_{2}(u) \prec \xi-a_{2}(u) \prec \xi$ can be handled easily via Lemma A.10. Of course, the first two terms in the equation above require to be treated as resonant terms. Note that modulo terms of order $\mathscr{C}_{T}^{3 \alpha-2}$ (or $\mathcal{E}^{\alpha / 2} \mathscr{C}^{2 \alpha-2}$ as defined in Lemma A.4) the terms $a_{1}(u) \circ \Delta u+a_{2}(u) \circ \xi$ are equivalent to

$$
a_{1}^{\prime}(u) \Pi_{\diamond}(a(u), \vartheta \circ \Delta \vartheta)+a_{2}^{\prime}(u)\left(\Pi_{\ll}(a(u), \vartheta) \circ \xi\right)
$$

and that by computations similar to those of Lemma 2.4 one can prove that

$$
\left(\Pi_{\ll}(a(u), \vartheta) \circ \xi\right)=\Pi_{\diamond}(a(u), \vartheta \circ \xi)+\mathscr{C}_{T}^{3 \alpha-2}
$$


so the resonant terms are comparable to the sum of the two terms

$$
a_{1}^{\prime}(u) \Pi_{\diamond}(a(u), \vartheta \circ \Delta \vartheta)+a_{2}^{\prime}(u) \Pi_{\diamond}(a(u), \vartheta \circ \xi)
$$

which require renormalisation of the form

$$
\frac{a_{1}^{\prime}(u) a_{2}(u)^{2}}{a_{1}(u)^{2}} \sigma_{\varepsilon}-\frac{a_{2}^{\prime}(u) a_{2}(u)}{a_{1}(u)} \sigma_{\varepsilon}
$$

and the convergence follows with the same arguments of Section 5.

We remark that the structure of the second renormalisation term, which is due to the r.h.s. in the equation, is the same of that found by Bailleul, Debussche and Hofmanová in Bailleul, Debussche and Hofmanová (2016).

REMARK 6.1. Our approach works straightforwardly for equation (8), namely

$$
\partial_{t} u(t, x)-a_{i j}(u(t, x)) \partial_{i j}^{2} u(t, x)=g(u(t, x)) \xi
$$

with $a: \mathbb{R} \rightarrow M_{2}(\mathbb{R})$ such that $\sum_{i, j} a(u)_{i j} x_{i} x_{j} \geq C|x|^{2} \forall x \in \mathbb{R}^{2}$ for $C>0$ and $\partial_{i j}^{2}:=\frac{\partial^{2}}{\partial x_{i} \partial x_{j}}$.

To see that, let $a(u):=\left(a_{i j}(u), g(u)\right) \in \mathbb{R}^{5}$ and $\eta=\left(\eta_{i, j}, \eta_{g}\right) \in \mathbb{R}^{5}$. Let $\mathscr{L}(\eta):=\partial_{t}-\eta_{i j} \partial_{i j}^{2}$ and $\Xi(\eta):=\eta_{g} \xi$ with the uniform ellipticity condition $\sum_{i, j} \eta_{i j} x_{i} x_{j} \geq C|x|^{2} \forall x \in \mathbb{R}^{2}$. It is easy to verify that Lemma 2.6 and Lemma 2.4 hold within this setting, just considering nonlinear paraproducts for functions depending on 5 parameters. We have then

$$
u=\Pi_{\ll}\left(a(u), \vartheta+U^{\sharp}+\mathscr{P} u_{0}^{\sharp}\right)
$$

with $\vartheta(\eta)$ stationary solution of $\mathscr{L} \vartheta(\eta)=\Xi(\eta), \mathscr{P}_{t} u_{0}^{\sharp}:=e^{\eta_{i j} \partial_{i j}^{2} t} u_{0}^{\sharp}$ and $U^{\sharp}(\eta)$, which solves

$$
\mathscr{L} U^{\sharp}(\eta)=\Pi_{\diamond}\left(\left(a(u), a^{\prime}(u)\right), \Theta_{1}\right)+\Pi_{\diamond}\left(\left(a(u), a^{\prime}(u)\right), \Theta_{2}\right)+Q\left(u, U^{\sharp}\right)
$$

with $Q\left(u, U^{\sharp}\right) \in \mathscr{C}^{2 \alpha-2-\varepsilon}, \Theta_{1} \in C_{\eta}^{2} C_{\eta^{\prime}}^{2} \mathscr{C}^{2 \alpha-2}=\vartheta(\eta) \circ \eta_{i j}^{\prime} \partial_{i j}^{2} \vartheta(\eta), \Theta_{2}\left(\eta, \eta^{\prime}\right)=$ $\vartheta(\eta) \circ \eta_{g}^{\prime} \xi$ and $U^{\sharp}(t=0)=0$. Note that we can write $\vartheta$ as

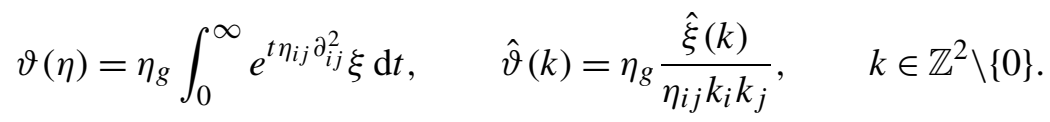

From the uniform ellipticity condition, we have $\|\vartheta\|_{C_{\eta}^{k} \mathscr{C}^{\alpha}} \lesssim\|\xi\|_{\mathscr{C}^{\alpha}}$, and Schauder estimates analogous to those of Lemma A.4 hold as well.

Now consider the renormalisation. We have

$$
\begin{aligned}
& H_{1}^{\varepsilon}\left(\eta, \eta^{\prime}\right):=\mathbb{E}\left(\Theta_{1}\left(\eta, \eta^{\prime}\right)\right)=-\eta_{g}^{2} \sum_{k \in \mathbb{Z}^{2} \backslash\{0\}} \hat{\psi}_{\varepsilon}(k)^{2} \frac{\sum_{i, j} \eta_{i j}^{\prime} k_{i} k_{j}}{\left(\sum_{i, j} \eta_{i j} k_{i} k_{j}\right)^{2}}, \\
& H_{2}^{\varepsilon}\left(\eta, \eta^{\prime}\right):=\mathbb{E}\left(\Theta_{2}\left(\eta, \eta^{\prime}\right)\right)=\eta_{g} \eta_{g}^{\prime} \sum_{k \in \mathbb{Z}^{2} \backslash\{0\}} \frac{\hat{\psi}_{\varepsilon}(k)^{2}}{\sum_{i, j} \eta_{i j} k_{i} k_{j}} .
\end{aligned}
$$


The convergence of $\Theta_{1}^{\varepsilon}-H_{1}^{\varepsilon}, \Theta_{2}^{\varepsilon}-H_{2}^{\varepsilon}$ in $C_{\left(\eta, \eta^{\prime}\right)}^{k} \mathscr{C}^{2 \alpha-2}\left(\mathbb{T}^{2}\right)$ can be obtained with the techniques used in Gubinelli, Imkeller and Perkowski (2015), Section 5.2.

7. Full generality. Within the framework of the present work, we are actually able to treat equations of the form (2), which if $a_{3}$ takes values in $[\lambda, 1]$ for some $\lambda>0$ is just

$$
\partial_{t} u(t, x)-a_{1}(u(t, x)) \Delta u(t, x)=\xi\left(a_{2}(u(t, x)), x\right),
$$

where $\xi\left(\eta_{2}, x\right)$ is a Gaussian process with covariance

$$
\mathbb{E}\left[\xi\left(\eta_{2}, x\right) \xi\left(\tilde{\eta}_{2}, \tilde{x}\right)\right]=F\left(\eta_{2}, \tilde{\eta}_{2}\right) \delta(x-\tilde{x}),
$$

where $F$ is a smooth covariance function. Let as before $2 / 3<\alpha<1$. In this case, we can take as a parametric equation

$$
\mathscr{L}(\eta) \vartheta:=\partial_{t} \vartheta(\eta, t, x)-\eta_{1} \Delta \vartheta(\eta, t, x)=\xi\left(\eta_{2}, x\right)
$$

whose solution $\vartheta$ is a Gaussian process, smooth with respect to the variable $\eta=\left(\eta_{1}, \eta_{2}\right)$ which we assume taking value in a compact subset of $\mathbb{R}^{2}$ for which $\eta_{1} \geq \lambda>0$ with fixed $\lambda$. Letting $a(u)=\left(a_{1}(u), a_{2}(u)\right)$ we can rewrite the 1.h.s. of equation (43) in the form

$$
\partial_{t} u-a_{1}(u) \Delta u=\Pi_{\diamond}(a(u), \mathscr{L} u)
$$

and the r.h.s. as

$$
\xi\left(a_{2}(u(t, x)), x\right)=\Pi_{\diamond}(a(u), \Xi),
$$

where $\Xi(\eta, x)=\xi\left(\eta_{2}, x\right)$. Now we perform the paraproduct decomposition to get

$$
\begin{aligned}
\Pi_{\prec}(a(u), \mathscr{L} u)-\Pi_{\prec}(a(u), \Xi)= & \Pi_{\diamond}(a(u), \Xi)+\Pi_{\circ}(a(u), \mathfrak{D} u) \\
& +\Pi_{\succ}(a(u), \Xi)+\Pi_{\succ}(a(u), \mathfrak{D} u) .
\end{aligned}
$$

We have introduced here the parametric differential operator $\mathfrak{D}(\eta):=\eta_{1} \Delta$ for $\eta=$ $\left(\eta_{1}, \eta_{2}\right)$

Let $\mathscr{P}_{t}(\eta):=e^{t \eta_{1} \Delta}$ as before, and invoke the paracontrolled Ansatz in the usual form

$$
u=\Pi_{\ll}\left(a(u), \vartheta+U^{\sharp}+\mathscr{P} u_{0}^{\sharp}\right) .
$$

Using that

$$
\begin{gathered}
\Pi_{\prec}\left(a(u), \mathscr{L} \Pi_{\ll}\left(a(u), \vartheta+U^{\sharp}+\mathscr{P} u_{0}^{\sharp}\right)\right) \\
=\Pi_{\ll}\left(a(u), \mathscr{L}\left(\vartheta+U^{\sharp}+\mathscr{P} u_{0}^{\sharp}\right)\right) \\
+\Psi\left(a(u), \vartheta+U^{\sharp}+\mathscr{P} u_{0}^{\sharp}\right)
\end{gathered}
$$


and observing that we can take $\mathscr{L} \vartheta=\Xi$ and that $\mathscr{L} \mathscr{P} u_{0}^{\sharp}=0$ to get

$$
\Pi_{\ll}\left(a(u), \mathscr{L} U^{\sharp}\right)=F\left(u, U^{\sharp}\right),
$$

where

$$
\begin{aligned}
F\left(u, U^{\sharp}\right)= & \Pi_{\circ}(a(u), \Xi)+\Pi_{\circ}(a(u), \mathfrak{D} u)+\Pi_{\succ}(a(u), \Xi)+\Pi_{\succ}(a(u), \mathfrak{D} u) \\
& +\left[\Pi_{\prec}(a(u), \Xi)-\Pi_{\gtrless}(a(u), \Xi)\right]-\Psi\left(a(u), \vartheta+U^{\sharp}+\mathscr{P} u_{0}^{\sharp}\right)
\end{aligned}
$$

which is solved by $U^{\sharp}$ satisfying

$$
\mathscr{L} U^{\sharp}=F\left(u, U^{\sharp}\right) .
$$

Indeed $\Pi_{\prec}\left(a(u), F\left(u, U^{\sharp}\right)\right)=F\left(u, U^{\sharp}\right)$, since $F\left(u, U^{\sharp}\right)$ does not depend on the additional parameter. Remark that the term $\Pi_{\prec}(a(u), \Xi)-\Pi_{\varkappa}(a(u), \Xi)$, which does not appear in the simpler case, can be treated with Lemma A.11.

It remains now to discuss the handling of the resonant products under the paracontrolled assumption, namely $\Pi_{\circ}(a(u), \Xi)$ and $\Pi_{\circ}(a(u), \mathfrak{D} u)$. The next lemma is a paralinearisation result adapted to our nonlinear context.

LEMMA 7.1. Assume that $u \in \mathscr{C}_{T}^{\rho}$ and $Z \in C_{\eta}^{2} \mathscr{C}_{T}^{\gamma}$ then if $\gamma+2 \rho>0$ we have

$$
C(u, Z):=\Pi_{\circ}(a(u), Z)-u \circ \Pi_{\prec}\left(\left(a(u), a^{\prime}(u)\right), \mathcal{D} Z\right) \in \mathscr{C}_{T}^{\gamma+2 \rho},
$$

where $\mathcal{D} Z\left(\left(\eta, \eta^{\prime}\right), t, x\right):=\sum_{i} \eta_{i}^{\prime} \partial_{\eta_{i}} Z(\eta, t, x)$.

PROOF.

$$
\begin{aligned}
\Pi_{\circ}(a & (u), Z)(t, x) \\
= & \sum_{i \sim j} \int_{y, z} K_{i, x}(y) K_{j, x}(z) Z(a(u(t, y)), t, z) \\
= & \sum_{i \sim j, k} \int_{\substack{y, z^{\prime} \\
z, z^{\prime \prime}}} K_{i, x}(y) K_{j, x}(z) P_{k, z}\left(z^{\prime \prime}\right) K_{k, z}\left(z^{\prime}\right) Z\left(a(u(t, y)), t, z^{\prime}\right) \\
= & \sum_{i \sim j, k} \int_{y, z^{\prime}} K_{i, x}(y) K_{j, x}(z) P_{k, z}\left(z^{\prime \prime}\right) K_{k, z}\left(z^{\prime}\right) \\
& \times\left[Z\left(a(u(t, y)), t, z^{\prime}\right)-Z\left(a\left(u\left(t, z^{\prime \prime}\right)\right), t, z^{\prime}\right)\right] \\
= & \sum_{i \sim j, k} \int_{y, z^{\prime}} K_{i, x}(y) K_{j, x}(z) P_{k, z}\left(z^{\prime \prime}\right) K_{k, z}\left(z^{\prime}\right) \\
& \times\left[\sum_{\ell} a_{\ell}^{\prime}\left(u\left(t, z^{\prime \prime}\right)\right) \delta u_{t z^{\prime \prime}}^{t y} \partial_{a_{\ell}} Z\left(a\left(u\left(t, z^{\prime \prime}\right)\right), t, z^{\prime}\right)\right]
\end{aligned}
$$




$$
\begin{aligned}
& +\sum_{\substack{i \sim j \\
k \sim j \\
k \sim j, z^{\prime \prime}}} K_{i, x}(y) K_{j, x}(z) P_{k, z}\left(z^{\prime \prime}\right) K_{k, z}\left(z^{\prime}\right) \\
& \times O\left(\left(\delta u_{t z^{\prime \prime}}^{t y}\right)^{2}\right) \partial_{\eta}^{2} Z\left(a(u(t, y)), t, z^{\prime}\right)
\end{aligned}
$$

and observe that the first term is equal to $u \circ \Pi_{\prec}\left(\left(a(u), a^{\prime}(u)\right), \mathcal{D} Z\right)$ while the second term can be easily estimated in $\mathscr{C}_{T}^{\gamma+2 \rho}$.

Using this result and Lemma A.11, we can expand in the same way as done for Lemma 2.4 to obtain

$$
\begin{aligned}
\Pi_{\circ}(a(u), \Xi) & =u \circ \Pi_{\prec}\left(\left(a(u), a^{\prime}(u)\right), \mathcal{D} \Xi\right)+\mathscr{C}_{T}^{3 \alpha-2} \\
& =\Pi_{\prec}(a(u), \vartheta) \circ \Pi_{\prec}\left(\left(a(u), a^{\prime}(u)\right), \mathcal{D} \Xi\right)+\mathscr{C}_{T}^{3 \alpha-2} \\
& =\Pi_{\diamond}\left(\left(a(u), a^{\prime}(u)\right), \vartheta \circ \mathcal{D} \Xi\right)+\mathscr{C}_{T}^{3 \alpha-2} .
\end{aligned}
$$

Similarly, noting that

$$
\begin{aligned}
& \Pi_{\prec}\left(\left(a(u), a^{\prime}(u)\right),(\mathcal{D} \mathfrak{D}) u\right) \\
& \quad=\Pi_{\prec}\left(\left(a(u), a^{\prime}(u)\right),(\mathcal{D} \mathfrak{D}) \Pi_{\ll}(a(u), \vartheta)\right)+\mathscr{C}_{T}^{3 \alpha-2} \\
& \quad=\Pi_{\prec}\left(\left(a(u), a^{\prime}(u)\right),(\mathcal{D} \mathfrak{D}) \vartheta\right)+\mathscr{C}_{T}^{3 \alpha-2},
\end{aligned}
$$

where $(\mathcal{D} \mathfrak{D})\left(\eta, \eta^{\prime}\right)=\eta_{1}^{\prime} \Delta$, we have by a straightforward generalisation of Lemma 2.4:

$$
\begin{aligned}
\Pi_{\circ}(a(u), \mathscr{L} u) & =u \circ \Pi_{\prec}\left(\left(a(u), a^{\prime}(u)\right),(\mathcal{D} \mathfrak{D}) u\right)+\mathscr{C}_{T}^{3 \alpha-2} \\
& =\Pi_{\prec}(a(u), \vartheta) \circ \Pi_{\prec}\left(\left(a(u), a^{\prime}(u)\right),(\mathcal{D} \mathfrak{D}) \vartheta\right)+\mathscr{C}_{T}^{3 \alpha-2} \\
& =\Pi_{\diamond}\left(\left(a(u), a^{\prime}(u)\right), \vartheta \circ(\mathcal{D D}) \vartheta\right)+\mathscr{C}_{T}^{3 \alpha-2} .
\end{aligned}
$$

Finally, the equation for $U^{\sharp}$ reads

$$
\mathscr{L} U^{\sharp}=\Pi_{\diamond}\left(\left(a(u), a^{\prime}(u)\right), \vartheta \circ \mathcal{D} \Xi+\vartheta \circ(\mathcal{D} \mathfrak{D}) \vartheta\right)+\mathscr{C}_{T}^{3 \alpha-2} .
$$

This can be solved essentially as we did in the simpler context. We see that the general enhancement has the form

$$
(\xi, \vartheta \circ \mathcal{D} \Xi+\vartheta \circ(\mathcal{D} \mathfrak{D}) \vartheta)
$$

which of course will require renormalisation like we did before. In particular,

$$
\begin{aligned}
(\vartheta \circ \mathcal{D} \Xi+\vartheta \circ(\mathcal{D D}) \vartheta)\left(\eta, \eta^{\prime}\right)= & \vartheta(\eta) \circ \eta_{2}^{\prime} \partial_{\eta_{2}} \xi\left(\eta_{2}, \cdot\right)+\vartheta(\eta) \circ \eta_{1}^{\prime} \Delta \vartheta(\eta) \\
= & -\frac{\eta_{2}^{\prime}}{\eta_{1}}\left(\Delta^{-1} \xi\left(\eta_{2}, \cdot\right)\right) \circ \partial_{\eta_{2}} \xi\left(\eta_{2}, \cdot\right) \\
& +\frac{\eta_{1}^{\prime}}{\eta_{1}^{2}}\left(\Delta^{-1} \xi\left(\eta_{2}, \cdot\right)\right) \circ \xi\left(\eta_{2}, \cdot\right),
\end{aligned}
$$


where we used that $\eta_{1} \Delta \vartheta(\eta)=-\xi\left(\eta_{2}, \cdot\right)$. Now observe that

$$
\mathbb{E}\left[\left(\Delta^{-1} \xi_{\varepsilon}\left(\eta_{2}, \cdot\right)\right) \circ \xi_{\varepsilon}\left(\eta_{2}, \cdot\right)\right]=-F\left(\eta_{2}, \eta_{2}\right) \sigma_{\varepsilon}
$$

and that

$$
\mathbb{E}\left[\left(\Delta^{-1} \xi_{\varepsilon}\left(\eta_{2}, \cdot\right)\right) \circ \partial_{\eta_{2}} \xi_{\varepsilon}\left(\eta_{2}, \cdot\right)\right]=-\left(\partial_{1} F\right)\left(\eta_{2}, \eta_{2}\right) \sigma_{\varepsilon}
$$

with $\partial_{1} F$ denoting the derivative with respect to the first entry.

In the end, the renormalised enhanced noise is obtained as the limit in $\mathcal{X}^{\alpha}$ of $\left(\xi_{\varepsilon}, \Xi_{2, \varepsilon}\right)$ where

$$
\begin{aligned}
\Xi_{2, \varepsilon}\left(\eta, \eta^{\prime}\right)= & -\frac{\eta_{2}^{\prime}}{\eta_{1}}\left(\Delta^{-1} \xi_{\varepsilon}\left(\eta_{2}, \cdot\right)\right) \circ \partial_{\eta_{2}} \xi_{\varepsilon}\left(\eta_{2}, \cdot\right) \\
& +\frac{\eta_{1}^{\prime}}{\eta_{1}^{2}}\left(\Delta^{-1} \xi_{\varepsilon}\left(\eta_{2}, \cdot\right)\right) \circ \xi_{\varepsilon}\left(\eta_{2}, \cdot\right)-H_{\varepsilon}\left(\eta, \eta^{\prime}\right)
\end{aligned}
$$

with

$$
H_{\varepsilon}\left(\eta, \eta^{\prime}\right)=\frac{\eta_{2}^{\prime}}{\eta_{1}}\left(\partial_{1} F\right)\left(\eta_{2}, \eta_{2}\right) \sigma_{\varepsilon}-\frac{\eta_{1}^{\prime}}{\eta_{1}^{2}} F\left(\eta_{2}, \eta_{2}\right) \sigma_{\varepsilon} .
$$

We remark that if we take $F\left(\eta_{2}, \tilde{\eta}_{2}\right)=\eta_{2} \tilde{\eta}_{2}$ we obtain again the situation treated in Section 6. Indeed in this case

$$
\Pi_{\diamond}\left(\left(a(u), a^{\prime}(u)\right), H_{\varepsilon}\right)=\frac{a_{2}^{\prime}(u) a_{2}(u)}{a_{1}(u)} \sigma_{\varepsilon}-\frac{a_{1}^{\prime}(u) a_{2}(u)^{2}}{a_{1}(u)^{2}} \sigma_{\varepsilon}
$$

which coincides with (42).

REMARK 7.2. Consider the more general equation (2), where the noise depends explicitly on time, for example, with a covariance

$$
\mathbb{E}\left[\xi(\eta, t, x) \xi\left(\eta^{\prime}, t^{\prime}, x^{\prime}\right)\right]=F\left(\eta, \eta^{\prime}\right) Q\left(t-t^{\prime}, x-x^{\prime}\right)
$$

with $F$ a smooth function and $Q$ a distribution of parabolic regularity $\rho>-4 / 3$. First note that the coefficient $a_{1}(u) \in[\lambda, 1]$ in front of the time derivative can be eliminated trivially by dividing.

In order to handle the time dependence of the noise, the framework of this paper will still apply, provided we consider space-time paraproducts instead of paraproducts which act only on the space variable. This can be done exactly following the lines of the paper Gubinelli, Imkeller and Perkowski (2015), where time paraproducts are employed in the paracontrolled approach to solutions to SDE driven by Gaussian signals.

The constraint of regularity $\rho>-4 / 3$ does allow to treat a noise which is white in time and smooth in space, but not a space-time white noise. It is well known that the first-order paracontrolled approach on which the present paper is based does not allow to treat this kind of irregular signals in full generality. 


\section{APPENDIX: BESOV SPACES AND LINEAR PARAPRODUCTS}

In this Appendix, we collect some classical results from harmonic analysis needed in the paper. For a gentle introduction to Littlewood-Paley theory and Besov spaces, see the recent monograph [Bahouri, Chemin and Danchin (2011)], where most of our results are taken from. There the case of tempered distributions on $\mathbb{R}^{d}$ is considered. The Schauder estimates for the heat semigroup (Lemma A.4) are classical and can be found in Gubinelli, Imkeller and Perkowski (2015), Gubinelli and Perkowski (2015).

Fix $d \in \mathbb{N}$ and denote by $\mathbb{T}^{d}=(\mathbb{R} /(2 \pi \mathbb{Z}))^{d}$ the $d$-dimensional torus. We focus here on distributions and SPDEs on the torus, but everything in this Appendix applies mutatis-mutandis on the full space $\mathbb{R}^{d}$; see Gubinelli, Imkeller and Perkowski (2015). The space of distributions $\mathscr{D}^{\prime}=\mathscr{D}^{\prime}\left(\mathbb{T}^{d}\right)$ is defined as the set of linear maps $f$ from $C^{\infty}=C^{\infty}\left(\mathbb{T}^{d}, \mathbb{C}\right)$ to $\mathbb{C}$, such that there exist $k \in \mathbb{N}$ and $C>0$ with

$$
|\langle f, \varphi\rangle|:=|f(\varphi)| \leq C \sup _{|\mu| \leq k}\left\|\partial^{\mu} \varphi\right\|_{L^{\infty}\left(\mathbb{T}^{d}\right)}
$$

for all $\varphi \in C^{\infty}$. In particular, the Fourier transform $\mathscr{F} f: \mathbb{Z}^{d} \rightarrow \mathbb{C}, \mathscr{F} f(k)=$ $\left\langle f, e^{-i k \cdot}\right\rangle$, is defined for all $f \in \mathscr{D}^{\prime}$, and it satisfies $|\mathscr{F} f(k)| \leq|P(k)|$ for a suitable polynomial $P$. We will also write $\hat{f}(k)=\mathscr{F} f(k)$. Conversely, if $(g(k))_{k \in \mathbb{Z}^{d}}$ is at most of polynomial growth, then its inverse Fourier transform

$$
\mathscr{F}^{-1} g=(2 \pi)^{-d} \sum_{k \in \mathbb{Z}^{d}} e^{i\langle k \cdot\rangle} g(k)
$$

defines a distribution, and we have $\mathscr{F}^{-1} \mathscr{F} f=f$ as well as $\mathscr{F} \mathscr{F}^{-1} g=g$. To see this, it suffices to note that the Fourier transform of $\varphi \in C^{\infty}$ decays faster than any rational function (we say that it is of rapid decay). Indeed, for $\mu \in \mathbb{N}_{0}^{d}$ we have $\left|k^{\mu} \hat{g}(k)\right|=\left|\mathscr{F}\left(\partial^{\mu} g\right)(k)\right| \leq\left\|\partial^{\mu} g\right\|_{L^{1}\left(\mathbb{T}^{d}\right)}$ for all $k \in \mathbb{Z}^{d}$. As a consequence, we get the Parseval formula $\langle f, \bar{\varphi}\rangle=(2 \pi)^{-d} \sum_{k} \hat{f}(k) \overline{\hat{\varphi}(k)}$ for $f \in \mathscr{D}^{\prime}$ and $\varphi \in C^{\infty}$.

Linear maps on $\mathscr{D}^{\prime}$ can be defined by duality: if $A: C^{\infty} \rightarrow C^{\infty}$ is such that for all $k \in \mathbb{N}$ there exists $n \in \mathbb{N}$ and $C>0$ with $\sup _{|\mu| \leq k}\left\|\partial^{\mu}(A \varphi)\right\|_{L^{\infty}} \leq$ $C \sup _{|\mu| \leq n}\left\|\partial^{\mu} \varphi\right\|$, then we set $\left\langle{ }^{t} A f, \varphi\right\rangle=\langle f, A \varphi\rangle$. Differential operators are defined by $\left\langle\partial^{\mu} f, \varphi\right\rangle=(-1)^{|\mu|}\left\langle f, \partial^{\mu} \varphi\right\rangle$. If $\varphi: \mathbb{Z}^{d} \rightarrow \mathbb{C}$ grows at most polynomially, then it defines a Fourier multiplier

$$
\varphi(\mathrm{D}) f=\mathscr{F}^{-1}(\varphi \mathscr{F} f),
$$

which gives us a distribution $\varphi(\mathrm{D}) f \in \mathscr{D}^{\prime}$ for every $f \in \mathscr{D}^{\prime}$.

Littlewood-Paley blocks give a decomposition of any distribution on $\mathscr{D}^{\prime}$ into an infinite series of smooth functions.

DEFINITION A.1. A dyadic partition of unity consists of two nonnegative radial functions $\chi, \rho \in C^{\infty}\left(\mathbb{R}^{d}, \mathbb{R}\right)$, where $\rho$ is supported in a ball $\mathscr{B}=\{|x| \leq c\}$ and $\rho$ is supported in an annulus $\mathscr{A}=\{a \leq|x| \leq b\}$ for suitable $a, b, c>0$, such that: 
1. $\chi+\sum_{j \geq 0} \rho\left(2^{-j}\right) \equiv 1$ and

2. $\chi \rho\left(2^{-j} \cdot\right) \equiv 0$ for $j \geq 1$ and $\rho\left(2^{-i}\right.$.) $\rho\left(2^{-j}\right.$. $) \equiv 0$ for all $i, j \geq 0$ with $\mid i-$ $j \mid>1$.

We will often write $\rho_{-1}=\chi$ and $\rho_{j}=\rho\left(2^{-j} \cdot\right)$ for $j \geq 0$.

Dyadic partitions of unity exist [Bahouri, Chemin and Danchin (2011)]. The reason for considering smooth partitions rather than indicator functions is that indicator functions do not have good Fourier properties. We fix a dyadic partition of unity $(\chi, \rho)$ and define the dyadic blocks

$$
\Delta_{j} f=\rho_{j}(\mathrm{D}) f=\mathscr{F}^{-1}\left(\rho_{j} \hat{f}\right), \quad j \geq-1 .
$$

We also use the notation

$$
S_{j} f=\sum_{i<j-1} \Delta_{i} f
$$

and notice that

$$
\begin{aligned}
\Delta_{j} f(x) & =\int K_{j, x}(y) f(y) \mathrm{d} y, \\
S_{j} f(x) & =\int P_{j, x}(y) f(y) \mathrm{d} y
\end{aligned}
$$

with $K_{j, x}(y)=2^{d j} K\left(2^{j}(x-y)\right), P_{j, x}(y)=\sum_{i<j-1} K_{i, x}(y), K$ radial with zero mean.

Every dyadic block has a compactly supported Fourier transform and, therefore, it belongs to $C^{\infty}$. It is easy to see that $f=\sum_{j \geq-1} \Delta_{j} f=\lim _{j \rightarrow \infty} S_{j} f$ for all $f \in \mathscr{D}^{\prime}$.

For $\alpha \in \mathbb{R}$, the Hölder-Besov space $\mathscr{C}^{\alpha}$ is given by $\mathscr{C}^{\alpha}=B_{\infty, \infty}^{\alpha}\left(\mathbb{T}^{d}, \mathbb{R}\right)$, where for $p, q \in[1, \infty]$ we define

$$
\begin{aligned}
B_{p, q}^{\alpha} & =B_{p, q}^{\alpha}\left(\mathbb{T}^{d}, \mathbb{R}\right) \\
& =\left\{f \in \mathscr{D}^{\prime}:\|f\|_{B_{p, q}^{\alpha}}=\left(\sum_{j \geq-1}\left(2^{j \alpha}\left\|\Delta_{j} f\right\|_{L^{p}}\right)^{q}\right)^{1 / q}<\infty\right\},
\end{aligned}
$$

with the usual interpretation as $\ell^{\infty}$ norm in case $q=\infty$. Then $B_{p, q}^{\alpha}$ is a Banach space and while the norm $\|\cdot\|_{B_{p, q}^{\alpha}}^{\alpha}$ depends on $(\chi, \rho)$, the space $B_{p, q}^{\alpha}$ does not, and any other dyadic partition of unity corresponds to an equivalent norm.

If $\alpha \in(0, \infty) \backslash \mathbb{N}$, then $\mathscr{C}^{\alpha}$ is the space of $\lfloor\alpha\rfloor$ times differentiable functions whose partial derivatives of order $\lfloor\alpha\rfloor$ are $(\alpha-\lfloor\alpha\rfloor)$-Hölder continuous [see p. 99 of Bahouri, Chemin and Danchin (2011)]. Note, however, that for $k \in \mathbb{N}$ the space $\mathscr{C}^{k}$ is strictly larger than $C^{k}$, the space of $k$ times continuously differentiable functions.

The following lemma gives useful characterisation of Besov regularity for functions that can be decomposed into pieces which are localised in Fourier space. 
Lemma A.2. 1. Let $\mathscr{A}$ be an annulus, let $\alpha \in \mathbb{R}$, and let $\left(u_{j}\right)$ be a sequence of smooth functions such that $\mathscr{F} u_{j}$ has its support in $2^{j} \mathscr{A}$, and such that $\left\|u_{j}\right\|_{L^{\infty}} \lesssim 2^{-j \alpha}$ for all $j$. Then

$$
u=\sum_{j \geq-1} u_{j} \in \mathscr{C}^{\alpha} \quad \text { and } \quad\|u\|_{\alpha} \lesssim \sup _{j \geq-1}\left\{2^{j \alpha}\left\|u_{j}\right\|_{L^{\infty}}\right\} .
$$

2. Let $\mathscr{B}$ be a ball, let $\alpha>0$, and let $\left(u_{j}\right)$ be a sequence of smooth functions such that $\mathscr{F} u_{j}$ has its support in $2^{j} \mathscr{B}$, and such that $\left\|u_{j}\right\|_{L^{\infty}} \lesssim 2^{-j \alpha}$ for all $j$. Then

$$
u=\sum_{j \geq-1} u_{j} \in \mathscr{C}^{\alpha} \quad \text { and } \quad\|u\|_{\alpha} \lesssim \sup _{j \geq-1}\left\{2^{j \alpha}\left\|u_{j}\right\|_{L^{\infty}}\right\}
$$

The Bernstein inequalities of the next lemma are extremely useful when dealing with functions with compactly supported Fourier transform.

Lemma A.3. Let $\mathscr{A}$ be an annulus and let $\mathscr{B}$ be a ball. For any $k \in \mathbb{N}_{0}$, $\lambda>0$, and $1 \leq p \leq q \leq \infty$ we have that

(i) if $u \in L^{p}$ is such that $\operatorname{supp}(\mathscr{F} u) \subseteq \lambda \mathscr{B}$, then

$$
\max _{\mu \in \mathbb{N}^{d}:|\mu|=k}\left\|\partial^{\mu} u\right\|_{L^{q}} \lesssim_{k} \lambda^{k+d\left(\frac{1}{p}-\frac{1}{q}\right)}\|u\|_{L^{p}}
$$

(ii) if $u \in L^{p}$ is such that $\operatorname{supp}(\mathscr{F} u) \subseteq \lambda \mathscr{A}$, then

$$
\lambda^{k}\|u\|_{L^{p}} \lesssim_{k \in \mathbb{N}^{d}:|\mu|=k}\left\|\partial^{\mu} u\right\|_{L^{p}} .
$$

We recall some standard heat kernel estimations [see Bahouri, Chemin and Danchin (2011), Chapter 2, and Gubinelli, Imkeller and Perkowski (2015), Gubinelli and Perkowski (2015)].

LEMmA A.4 (Schauder estimates). Let $V_{t}=\int_{0}^{t} e^{\eta(t-s) \Delta} v_{s} \mathrm{~d} s$ and $\mathscr{P}_{t} u_{0}=$ $e^{\eta \Delta t} u_{0}$, with $\eta \geq \lambda$. We define $\mathscr{L}_{T}^{\alpha}$ and $C_{\eta}^{k} \mathscr{L}_{T}^{\alpha}, C_{\eta}^{k} \mathscr{C}_{T}^{\alpha}$ for $k \in \mathbb{N}$ as in (9), (10) and introduce the norm

$$
\|v\|_{\mathcal{E}_{T}^{\delta} \mathscr{C}^{\alpha}}=\sup _{t \in[0, T]} t^{\delta}\|v(t, \cdot)\|_{\mathscr{C}^{\alpha}} .
$$

Then for any $\gamma \in[0,1)$ and $\alpha \in \mathbb{R}$ :

$$
\begin{aligned}
& \sup _{t \in[0, T]} t^{\gamma}\left\|V_{t}\right\|_{C_{\eta}^{k \mathscr{C}} \mathscr{C}^{\alpha-2 \beta}} \lesssim \sup _{t \in[0, T]} t^{\gamma+\beta}\left\|v_{t}\right\|_{\mathscr{C}^{\alpha-2}} \quad \forall \beta \in[0,1), \\
&\|V\|_{C_{\eta}^{k} \mathscr{L}_{T}^{\alpha-2 \beta}} \lesssim T^{\beta}\|v\|_{\mathscr{C}_{T}^{\alpha-2}} \quad \forall \beta \in[0,1), \\
&\|V\|_{C_{\eta}^{k} \mathscr{L}_{T}^{\alpha-2 \beta}} \lesssim T^{\beta-\delta}\|v\|_{\mathcal{E}_{T}^{\delta} \mathscr{C}^{\alpha-2}} \quad \forall \beta \in[0,1), \forall \delta \in[0, \beta],
\end{aligned}
$$




$$
\begin{gathered}
\|V\|_{C_{\eta}^{k} \mathscr{L}_{T}^{\gamma}} \lesssim T^{\frac{\rho-\gamma}{2}+1-\delta}\|v\|_{\mathcal{E}_{T}^{\delta} \mathscr{C}^{\rho}} \\
\forall \rho \in[\gamma-2, \gamma), \forall \delta \in[0,(\rho-\gamma) / 2+1], \\
\left\|\mathscr{P}_{t} u_{0}\right\|_{C_{\eta}^{k} \mathscr{C}^{\alpha}} \lesssim t^{-\frac{\alpha-\beta}{2}}\left\|u_{0}\right\|_{\mathscr{C}^{\beta}} \quad \forall \beta<\alpha, \\
\left\|\mathscr{P}_{t} u_{0}-\mathscr{P}_{s} u_{0}\right\|_{C_{\eta}^{k} \mathscr{C}^{\alpha}} \lesssim s^{-\delta}|t-s|^{\frac{\beta-\alpha}{2}+\delta}\left\|u_{0}\right\|_{\mathscr{C}} \beta \\
\forall t \neq s \in \mathbb{R}^{+}, \beta \leq \alpha+2, \delta \in[0,1-(\beta-\alpha) / 2] .
\end{gathered}
$$

We need the following interpolation lemma.

LemmA A.5. Let $\gamma \in(0,2), 0<\varepsilon<\gamma$ and $u \in \mathscr{L}_{T}^{\gamma}$. Then

$$
\|u\|_{C_{T}^{\gamma / 2-\varepsilon / 2} L^{\infty}} \lesssim\|u\|_{\mathscr{L}_{T}^{\gamma}} \text {. }
$$

PROOF.

$$
\sup _{s \neq t} \frac{\left\|u_{t}-u_{s}\right\|_{L^{\infty}}}{|t-s|^{\gamma / 2-\varepsilon / 2}} \leq \sup _{s \neq t}\left[\sum_{i \leq n} \frac{\left\|\Delta_{i} u_{t}-\Delta_{i} u_{s}\right\|_{L^{\infty}}}{|t-s|^{\gamma / 2-\varepsilon / 2}}+\sum_{i>n} \frac{\left\|\Delta_{i} u_{t}-\Delta_{i} u_{s}\right\|_{L^{\infty}}}{|t-s|^{\gamma / 2-\varepsilon / 2}}\right]
$$

and choosing $2^{-n-1} \leq|t-s|^{1 / 2} \leq 2^{-n}$ we obtain

$$
\begin{aligned}
& \sum_{i<n} \frac{\left\|\Delta_{i} u_{t}-\Delta_{i} u_{s}\right\|_{L^{\infty}}}{|t-s|^{\gamma / 2-\varepsilon / 2}} \lesssim\|u\|_{C_{T}^{\gamma / 2} \mathscr{C}^{0}} \sum_{i \leq n}|t-s|^{\varepsilon / 2}, \\
& \sum_{i \geq n} \frac{\left\|\Delta_{i} u_{t}-\Delta_{i} u_{s}\right\|_{L^{\infty}}}{|t-s|^{\gamma / 2-\varepsilon / 2}} \lesssim\|u\|_{\mathscr{C}_{T}^{\gamma}} \sum_{i>n} 2^{-\gamma i} 2^{-(\gamma+\varepsilon) n}
\end{aligned}
$$

and this yields the result.

Terms of the type $\|a(u(t, x))\|_{\mathscr{C} 0}$ with $a: \mathbb{R} \rightarrow \mathbb{R}$ cannot be estimated directly with their Hölder norm. In the following lemma, we prove some bounds used in Section 4.

LeMMA A.6. Let $a \in C_{b}^{3}$ uniformly bounded and $u \in \mathscr{L}_{T}^{\alpha}=\mathscr{C}_{T}^{\alpha} \cap C_{T}^{\alpha / 2} \mathscr{C}^{0}$, then

$$
\begin{aligned}
&\|a(u)\|_{\mathscr{L}_{T}^{\alpha}} \lesssim\|a\|_{L^{\infty}}+\left\|a^{\prime}\right\|_{L^{\infty}}\left(\|u\|_{C_{T}^{\alpha / 2} \mathscr{C}^{0}}+\|u\|_{\mathscr{C}_{T}^{\alpha}}\right) \\
&+\left\|a^{\prime \prime}\right\|_{L^{\infty}}\|u\|_{C_{T}^{\alpha / 2} \mathscr{C}^{0}}\|u\|_{\mathscr{C}_{T}^{\alpha}} \\
& \lesssim+\|u\|_{\mathscr{L}_{T}^{\alpha}}+\|u\|_{\mathscr{L}_{T}^{\alpha}}^{2}, \\
&\left\|a\left(u_{1}\right)-a\left(u_{2}\right)\right\|_{\mathscr{L}_{T}^{\alpha}} \lesssim\left\|u_{1}-u_{2}\right\|_{\mathscr{L}_{T}^{\alpha}}\left(1+\left\|u_{1}\right\|_{\mathscr{C}_{T}^{\alpha}}+\left\|u_{2}\right\|_{\mathscr{C}_{T}^{\alpha}}\right)^{2}, \\
&\left\|a\left(u_{1}\right)-a\left(u_{2}\right)\right\|_{\mathscr{C}_{T}^{0}} \lesssim\left\|u_{1}-u_{2}\right\|_{\mathscr{C}_{T}^{0}}\left(1+\left\|u_{1}\right\|_{\mathscr{C}_{T}^{\alpha}}+\left\|u_{2}\right\|_{\mathscr{C}_{T}^{\alpha}}\right) .
\end{aligned}
$$


Proof. The bound on $\|a(u)\|_{\mathscr{C}_{T}^{\alpha}}$ is trivial. We estimate $\left\|a\left(u_{t}\right)-a\left(u_{s}\right)\right\|_{\mathscr{C}} 0$ as

$$
\begin{aligned}
& \left|\int_{z} K_{i, x}(z)[a(u(t, z))-a(u(s, z))]\right| \\
& =\left|\int_{z, \tau \in[0,1]} K_{i, x}(z) a^{\prime}\left(\delta_{\tau} u_{s z}^{t z}\right)[u(t, z)-u(s, z)]\right| \\
& \leq\left|\int_{z, w, \tau \in[0,1]} K_{i, x}(z) \sum_{j \lesssim k \sim i}\left[\Delta_{j} u(t, z)-\Delta_{j} u(s, z)\right] K_{k, z}(w) a^{\prime}\left(\delta_{\tau} u_{s w}^{t w}\right)\right| \\
& \quad+\left|\int_{z, w, \tau \in[0,1]} K_{i, x}(z) \sum_{k \lesssim j \sim i}\left[\Delta_{j} u(t, z)-\Delta_{j} u(s, z)\right] K_{k, z}(w) a^{\prime}\left(\delta_{\tau} u_{s w}^{t w}\right)\right| \\
& \quad+\left|\int_{z, w, \tau \in[0,1]} K_{i, x}(z) \sum_{k \sim j \gtrsim i}\left[\Delta_{j} u(t, z)-\Delta_{j} u(s, z)\right] K_{k, z}(w) a^{\prime}\left(\delta_{\tau} u_{s w}^{t w}\right)\right| .
\end{aligned}
$$

If $k>-1$, we have

$$
\int_{w} K_{k, z}(w) a^{\prime}\left(\delta_{\tau} u_{s w}^{t w}\right)=\int_{w} K_{k, z}(w)\left[a^{\prime}\left(\delta_{\tau} u_{s w}^{t w}\right)-a^{\prime}\left(\delta_{\tau} u_{s z}^{t z}\right)\right]
$$

and then the first term above becomes

$$
\begin{aligned}
& \mid \int_{z, w, \tau, \sigma} K_{i, x}(z) \sum_{j \lesssim k \sim i}\left[\Delta_{j} u(t, z)\right. \\
& \left.-\Delta_{j} u(s, z)\right] K_{k, z}(w) a^{\prime \prime}\left(\delta_{\sigma}\left(\delta_{\tau} u_{s}^{t}\right)_{z}^{w}\right)\left[\delta u_{t z}^{t w}-\delta u_{s z}^{s w}\right] \mid \\
& \quad \lesssim\left\|a^{\prime \prime}\right\|_{L^{\infty}}\left\|u_{t}-u_{s}\right\|_{\mathscr{C}^{0}} \sum_{j \lesssim k \sim i} \int_{w}\left|K_{k, z}(w)\right||w-z|^{\alpha}\|u\|_{\mathscr{C}_{T}^{\alpha}} \\
& \quad \lesssim i 2^{-\alpha i}\left\|a^{\prime \prime}\right\|_{L^{\infty}}\left\|u_{t}-u_{s}\right\|_{\mathscr{C}^{0}}\|u\|_{\mathscr{C}_{T}^{\alpha}} .
\end{aligned}
$$

The second term is

$$
\begin{aligned}
& \left|\int_{z, w, \tau \in[0,1]} K_{i, x}(z) \sum_{j \sim i}\left[\Delta_{j} u(t, \cdot)-\Delta_{j} u(s, \cdot)\right](z) P_{j, z}(w) a^{\prime}\left(\delta_{\tau} u_{s w}^{t w}\right)\right| \\
& \quad \lesssim\left\|u_{t}-u_{s}\right\|_{\mathscr{C}^{0}}\left\|P_{j, z}(w) a^{\prime}\left(\delta_{\tau} u_{s w}^{t w}\right)\right\|_{L^{\infty}} \lesssim\left\|u_{t}-u_{s}\right\|_{\mathscr{C}^{0}}\left\|a^{\prime}\right\|_{L^{\infty}} .
\end{aligned}
$$

The third term can be estimated as the first one when $k>-1$. Otherwise, we just bound it as

$$
\begin{aligned}
& \left|\int_{z, w, \tau \in[0,1]} K_{i, x}(z) \sum_{j \leq N}\left[\Delta_{j} u(t, \cdot)-\Delta_{j} u(s, \cdot)\right](z) K_{-1, z}(w) a^{\prime}\left(\delta_{\tau} u_{s w}^{t w}\right)\right| \\
& \quad \lesssim\left\|u_{t}-u_{s}\right\|_{\mathscr{C}^{0}}\left\|a^{\prime}\right\|_{L^{\infty}} .
\end{aligned}
$$


For the three terms together, we have the bound

$$
\left\|a\left(u_{t}\right)-a\left(u_{s}\right)\right\|_{\mathscr{C}^{0}} \lesssim\left\|u_{t}-u_{s}\right\|_{\mathscr{C}^{0}}\left(\left\|a^{\prime}\right\|_{L^{\infty}}+\left\|a^{\prime \prime}\right\|_{L^{\infty}}\|u\|_{\mathscr{C}_{T}^{\alpha}}\right) .
$$

With the same technique, we obtain

$$
\begin{aligned}
\left\|a\left(u_{1}\right)-a\left(u_{2}\right)\right\|_{C_{T}^{\alpha / 2} \mathscr{C}^{0}} \lesssim & \left\|a^{\prime}\right\|_{L^{\infty}}\left\|u_{1}-u_{2}\right\|_{C_{T}^{\alpha / 2} \mathscr{C}^{0}} \\
& +\left\|a^{\prime \prime}\right\|_{L^{\infty}}\left\|u_{1}-u_{2}\right\|_{C_{T}^{\alpha / 2} \mathscr{C} 0}\left\|u_{1}-u_{2}\right\|_{\mathscr{C}_{T}^{\alpha}}^{\alpha} \\
& +\left\|a^{\prime \prime \prime}\right\|_{L^{\infty}}\left\|u_{1}-u_{2}\right\|_{C_{T}^{\alpha / 2} \mathscr{C}_{0} 0}\left\|u_{1}-u_{2}\right\|_{\mathscr{C}_{T}^{\alpha}}^{2}
\end{aligned}
$$

and this gives the second estimate. The third one can be obtained easily.

A.1. Bony's paraproduct. In terms of Littlewood-Paley blocks, the product of two smooth functions $f g$ can be decomposed as

$$
f g=\sum_{j \geq-1} \sum_{i \geq-1} \Delta_{i} f \Delta_{j} g=f \prec g+f \succ g+f \circ g,
$$

where the paraproducts $f \prec g$ and $f \succ g$ and the resonant product $f \circ g$ are defined as

$$
f \prec g=g \succ f:=\sum_{j \geq-1} \sum_{i=-1}^{j-2} \Delta_{i} f \Delta_{j} g \text { and } f \circ g:=\sum_{|i-j| \leq 1} \Delta_{i} f \Delta_{j} g .
$$

We will often use the shortcuts $\sum_{i \sim j}$ for $\sum_{|i-j| \leq 1}$ and $\sum_{i \lesssim j}$ for $\sum_{i<j-1}$. Of course, the decomposition depends on the dyadic partition of unity used to define the blocks $\Delta_{j}$, and also on the particular choice of the pairs $(i, j)$ in the diagonal part. The choice of taking all $(i, j)$ with $|i-j| \leq 1$ into the diagonal part corresponds to property 2 of Definition A.1 (definition of dyadic partitions of unity).

Bony's crucial observation [Bony (1981), Meyer (1980)] is that the paraproduct $f \prec g$ (and thus $f \succ g$ ) is always a well-defined distribution. Heuristically, $f \prec g$ behaves at large frequencies like $g$ (and thus retains the same regularity), and $f$ provides only a frequency modulation of $g$. The only difficulty in constructing $f g$ for arbitrary distributions lies in handling the resonant product $f \circ g$. The basic result about these bilinear operations is given by the following estimates.

Theorem A.7 (Paraproduct estimates). For any $\beta \in \mathbb{R}$ and $f, g \in \mathscr{D}^{\prime}$, we have

$$
\|f \prec g\|_{\mathscr{C}^{\beta}} \lesssim \beta\|f\|_{L^{\infty}}\|g\|_{\mathscr{C} \beta},
$$

and for $\alpha<0$, furthermore,

$$
\|f \prec g\|_{\mathscr{C} \alpha+\beta} \lesssim \alpha, \beta\|f\|_{\mathscr{C}} \alpha\|g\|_{\mathscr{C} \beta} .
$$

For $\alpha+\beta>0$, we have

$$
\|f \circ g\|_{\mathscr{C} \alpha+\beta} \lesssim \alpha, \beta\|f\|_{\mathscr{C} \alpha}\|g\|_{\mathscr{C} \beta} .
$$


Bony proved also a basic paralinearisation result, soon after improved by Meyer. We give here a particular version suited to our purposes.

Theorem A.8. Let $\alpha \in(0,1), f \in\left(\mathscr{C}^{\alpha}\right)^{k}$ and $F \in C^{3}\left(\mathbb{R}^{k} ; \mathbb{R}\right)$ then

$$
R_{F}(f):=F(f)-F^{\prime}(f) \prec f \in \mathscr{C}^{2 \alpha}
$$

with

$$
\left\|R_{F}(f)\right\|_{\mathscr{C}^{2 \alpha}} \lesssim\|F\|_{C^{2}}\left(1+\|f\|_{\mathscr{C}^{\alpha}}\right)^{2} .
$$

Moreover, the map $f \mapsto R_{F}(f)$ is locally Lipshitz and

$$
\left\|R_{F}(f)-R_{F}(\tilde{f})\right\|_{\mathscr{C}^{2 \alpha}} \lesssim\|F\|_{C^{3}}\left(1+\|f\|_{\mathscr{C}^{\alpha}}+\|\tilde{f}\|_{\mathscr{C}^{\alpha}}\right)^{2}\|\tilde{f}-f\|_{\mathscr{C}^{\alpha}} .
$$

The additional key ingredient at the core of the paracontrolled approach is a commutation result proved in Gubinelli, Imkeller and Perkowski (2015), Lemma 2.4:

Lemma A.9. Assume that $\alpha, \beta, \gamma \in \mathbb{R}$ are such that $\alpha+\beta+\gamma>0$ and $\beta+$ $\gamma<0$. Then for $f, g, h \in C^{\infty}$, the trilinear operator

$$
C(f, g, h):=((f \prec g) \circ h)-f(g \circ h)
$$

allows for the bound

$$
\|C(f, g, h)\|_{\mathscr{C} \beta+\gamma} \lesssim\|f\|_{\mathscr{C} \alpha}\|g\|_{\mathscr{C} \beta}\|h\|_{\mathscr{C} \gamma},
$$

and can thus be uniquely extended to a bounded trilinear operator

$$
C: \mathscr{C}^{\alpha} \times \mathscr{C}^{\beta} \times \mathscr{C}^{\alpha} \rightarrow \mathscr{C}^{\beta+\gamma} .
$$

We will need the following two lemmas to compare standard and time-smoothed paraproducts. The first one has essentially the same proof as Gubinelli, Imkeller and Perkowski (2015), Lemma 5.1.

Lemma A.10. Let $\rho \in(0,2), \gamma \in \mathbb{R}$. Then for every $\varepsilon>0$ we have the bound

$$
\|g \prec h-g \ll h\|_{\mathscr{C}_{T}^{\rho+\gamma-\varepsilon}} \lesssim\|g\|_{C_{T}^{\rho / 2} \mathscr{C} 0}\|h\|_{\mathscr{C}_{T}^{\gamma}} .
$$

The second lemma has a standard proof.

Lemma A.11. Let $g \in \mathscr{L}_{T}^{\rho}, h \in C_{\eta}^{1} \mathscr{C}_{T}^{\gamma}$ with $\rho \in(0,1), \gamma \in \mathbb{R}$. We have, $\forall \varepsilon>0$

$$
\left\|\Pi_{\prec}(g, h)-\Pi_{\ll}(g, h)\right\|_{\mathscr{C}_{T}^{\rho+\gamma-\varepsilon}} \lesssim\|g\|_{\mathscr{L}_{T}^{\rho}}\|h\|_{C_{\eta}^{1} \mathscr{C}_{T}^{\gamma}}
$$




\section{REFERENCES}

AlinhaC, S. and GÉrArd, P. (2007). Pseudo-Differential Operators and the Nash-Moser Theorem. Graduate Studies in Mathematics 82. Amer. Math. Soc., Providence, RI. Translated from the 1991 French original by Stephen S. Wilson. MR2304160

Allez, R. and ChOuK, K. (2015). The continuous Anderson Hamiltonian in dimension two. Arxiv preprint. Available at arXiv:1511.02718.

Bahouri, H., Chemin, J.-Y. and Danchin, R. (2011). Fourier Analysis and Nonlinear Partial Differential Equations. Grundlehren der Mathematischen Wissenschaften [Fundamental Principles of Mathematical Sciences] 343. Springer, Heidelberg. MR2768550

BAILLEUL, I. (2015). Flows driven by rough paths. Rev. Mat. Iberoam. 31 901-934. MR3420480

BAILleul, I. and BERNicot, F. (2016a). Heat semigroup and singular PDEs. J. Funct. Anal. 270 3344-3452. MR3475460

BAILleul, I. and BERNiCOT, F. (2016b). Higher order paracontrolled calculus. Arxiv preprint. Available at arXiv:1609.06966.

BAilleul, I. and CATELlier, R. (2017). Rough flows and homogenization in stochastic turbulence. J. Differential Equations 263 4894-4928. MR3680942

Bailleul, I., Debussche, A. and Hofmanová, M. (2016). Quasilinear generalized parabolic Anderson model equation. Arxiv preprint. Available at arXiv:1610.06726.

BONY, J.-M. (1981). Calcul symbolique et propagation des singularités pour les équations aux dérivées partielles non linéaires. Ann. Sci. Éc. Norm. Super. (4) 14 209-246. MR0631751

BONY, J.-M. (1991). Analyse microlocale des équations aux dérivées partielles non linéaires. In Microlocal Analysis and Applications (Montecatini Terme, 1989). Lecture Notes in Math. 1495 1-45. Springer, Berlin. MR1178555

Catellier, R. and Chouk, K. (2013). Paracontrolled distributions and the 3-dimensional stochastic quantization equation. Arxiv preprint. Available at arXiv:1310.6869.

Catellier, R. and Gubinelli, M. (2016). Averaging along irregular curves and regularisation of ODEs. Stochastic Process. Appl. 126 2323-2366. MR3505229

FriZ, P. K. and HAIRER, M. (2014). A Course on Rough Paths. Springer, Cham. MR3289027

GERENCSÉR, M. and HAIRER, M. (2017). A solution theory for quasilinear singular SPDEs. Arxiv preprint. Available at arXiv:1712.01881.

Gubinelli, M. (2012). Rough solutions for the periodic Korteweg-de Vries equation. Commun. Pure Appl. Anal. 11 709-733. MR2861805

Gubinelli, M., Imkeller, P. and Perkowski, N. (2015). Paracontrolled distributions and singular PDEs. Forum Math. Pi 3 e6, 75. MR3406823

Gubinelli, M. and Perkowski, N. (2015). Lectures on Singular Stochastic PDEs. Ensaios Matemáticos [Mathematical Surveys] 29. Sociedade Brasileira de Matemática, Rio de Janeiro. MR3445609

Gubinelli, M. and Perkowski, N. (2017). KPZ reloaded. Comm. Math. Phys. 349 165-269. MR3592748

HAIRER, M. (2013). Solving the KPZ equation. Ann. of Math. (2) 178 559-664. MR3071506

HAIRER, M. (2014a). A theory of regularity structures. Invent. Math. 198 269-504. MR3274562

HAIRER, M. (2014b). Singular stochastic PDEs. In Proceedings of the International Congress of Mathematicians-Seoul 2014. Vol. IV 49-73. Kyung Moon Sa, Seoul. MR3727602

HAIRER, M. and QUASTEL, J. (2015). A class of growth models rescaling to KPZ. Arxiv preprint. Available at arXiv:1512.07845.

Hairer, M. and Shen, H. (2016). The dynamical sine-Gordon model. Comm. Math. Phys. 341 933-989. MR3452276

HAIRER, M. and XU, W. (2016). Large scale behaviour of 3D phase coexistence models. Arxiv preprint. Available at arXiv:1601.05138. 
Hörmander, L. (1990). The Nash-Moser theorem and paradifferential operators. In Analysis, et Cetera 429-449. Academic Press, Boston, MA. MR1039355

HU, Y. and LÊ, K. (2017). Nonlinear Young integrals and differential systems in Hölder media. Trans. Amer. Math. Soc. 369 1935-2002. MR3581224

Kelly, D. and Melbourne, I. (2017). Deterministic homogenization for fast-slow systems with chaotic noise. J. Funct. Anal. 272 4063-4102. MR3626033

KunitA, H. (1984). Stochastic differential equations and stochastic flows of diffeomorphisms. In École d'Été de Probabilités de Saint-Flour, XII-1982. Lecture Notes in Math. 1097 143-303. Springer, Berlin. MR0876080

LYONS, T. J. (1998). Differential equations driven by rough signals. Rev. Mat. Iberoam. 14 215-310. MR1654527

Lyons, T. J., Caruana, M. and LÉvy, T. (2007). Differential Equations Driven by Rough Paths. Lecture Notes in Math. 1908. Springer, Berlin. MR2314753

Lyons, T. and Qian, Z. (2003). System Control and Rough Paths, 1st ed. Clarendon Press, Oxford.

Meyer, Y. (1980). Remarques sur un théorème de J.-M. Bony. Prepublications Mathematiques D'Orsay 2546.

Mourrat, J.-C. and Weber, H. (2017). The dynamic $\Phi_{3}^{4}$ model comes down from infinity. Comm. Math. Phys. 356 673-753. MR3719541

OTTO, F. and WeBER, H. (2016). Quasilinear SPDEs via rough paths. Arxiv preprint. Available at arXiv:1605.09744.

CEREMADE

PARIS-DAUPHINE UNIVERSITY

PARIS

FRANCE

E-MAIL: furlan@ceremade.dauphine.fr
IAM \& HCM

BONN UNIVERSITY

BONN

GERMANY

E-MAIL: gubinelli@iam.uni-bonn.de 